\title{
CAMKII suppresses an efferocytosis pathway in macrophages and promotes atherosclerotic plaque necrosis
}

\author{
Amanda C. Doran, ${ }^{1}$ Lale Ozcan, ${ }^{1}$ Bishuang Cai, ${ }^{1}$ Ze Zheng, ${ }^{1}$ Gabrielle Fredman, ${ }^{2}$ Christina C. Rymond, ${ }^{1}$ Bernhard Dorweiler, ${ }^{3}$ \\ Judith C. Sluimer, ${ }^{4}$ Joanne Hsieh, ${ }^{1}$ George Kuriakose, ${ }^{5}$ Alan R. Tall, ${ }^{1}$ and Ira Tabas ${ }^{1,5,6}$ \\ 'Department of Medicine, Columbia University, New York, New York, USA. ²Department of Molecular and Cellular Physiology, Center for Cardiovascular Sciences, Albany Medical Center, Albany, New York, \\ USA. ${ }^{3}$ Department of Cardiothoracic and Vascular Surgery, Universitätsmedizin Mainz, Johannes-Gutenberg University, Mainz, Germany. ${ }^{4}$ Department of Pathology, Maastricht University Medical Center, \\ Maastricht, The Netherlands. ${ }^{5}$ Department of Physiology and Cellular Biophysics and ${ }^{6}$ Department of Pathology and Cell Biology, Columbia University, New York, New York, USA.
}

\begin{abstract}
Atherosclerosis is the underlying etiology of cardiovascular disease, the leading cause of death worldwide. Atherosclerosis is a heterogeneous disease in which only a small fraction of lesions lead to heart attack, stroke, or sudden cardiac death. A distinct type of plaque containing large necrotic cores with thin fibrous caps often precipitates these acute events. Here, we

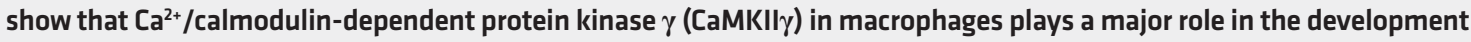
of necrotic, thin-capped plaques. Macrophages in necrotic and symptomatic atherosclerotic plaques in humans as well as advanced atherosclerotic lesions in mice demonstrated activation of CaMKII. Western diet-fed LDL receptor-deficient ( ddIr $^{-/-}$) mice with myeloid-specific deletion of CaMKII had smaller necrotic cores with concomitantly thicker collagen caps. These lesions demonstrated evidence of enhanced efferocytosis, which was associated with increased expression of the macrophage efferocytosis receptor MerTK. Mechanistic studies revealed that CaMKIl $\gamma$-deficient macrophages and atherosclerotic lesions lacking myeloid CaMKIl $\gamma$ had increased expression of the transcription factor ATF6. We determined that ATF6 induces liver X receptor- $\alpha(\mathrm{LXR} \alpha)$, an Mertk-inducing transcription factor, and that increased MerTK expression and efferocytosis in CaMKIl $\gamma$ deficient macrophages is dependent on LXR $\alpha$. These findings identify a macrophage CaMKIl $\gamma /$ ATF6/LXR $\alpha /$ MerTK pathway as a key factor in the development of necrotic atherosclerotic plaques.
\end{abstract}

\section{Introduction}

Despite major medical advances in the past 50 years, cardiovascular disease remains the major cause of morbidity and mortality worldwide (1). Ischemic cardiovascular disease is caused by atherosclerosis, a lipoprotein-driven inflammatory process in which lipid, macrophages and other immune cells, and fibrotic elements accumulate in the intima of large arteries at sites of disturbed blood flow $(2,3)$. While most atherosclerotic plaques are clinically silent, a subset of lesions can trigger thrombotic vascular occlusion, leading to unstable angina, myocardial infarction, stroke, or sudden cardiac death. Two morphologically distinct lesion types can lead to occlusive thrombosis: rupture-prone plaques with necrotic cores and thin fibrous caps (thin-cap fibroatheroma [TCFA]) and fibrous plaques with superficial endothelial erosion $(4,5)$. Given the clinical importance of TCFA, understanding the processes that contribute to the development of the necrotic core is essential.

In normal physiology, apoptotic cells are rapidly and efficiently cleared by phagocytes through efferocytosis, which is the receptor-mediated process by which macrophages and oth-

Authorship note: A.C. Doran and L. Ozcan contributed equally to this work. Conflict of interest: The authors have declared that no conflict of interest exists. Submitted: April 24, 2017; Accepted: August 10, 2017.

Reference information: / Clin Invest. 2017;127(11):4075-4089.

https://doi.org/10.1172/JCI94735. er phagocytes recognize, engulf, and degrade apoptotic cells. When efferocytosis fails, dead cells undergo secondary necrosis and generate an intense inflammatory response (6). Studies have shown that macrophage-mediated efferocytosis is defective in advanced human plaques that have not yet ruptured $(7,8)$. Further, experiments using genetically engineered mice, i.e., mice that lack the key macrophage efferocytosis receptor MerTK, have demonstrated a causal relationship between defective macrophage-mediated efferocytosis and plaque necrosis $(9,10)$. Recently, our group has demonstrated that MerTK expression is decreased in symptomatic carotid artery lesions as compared with asymptomatic lesions (9). The mechanisms that contribute to defective efferocytosis in advanced atherosclerosis, however, have not yet been fully elucidated.

$\mathrm{Ca}^{2+} /$ calmodulin-dependent protein kinase II (CaMKII) is a serine-threonine kinase that is a key mediator of $\mathrm{Ca}^{2+}$-regulated processes in many different cell types $(11,12)$. There are 4 CaMKII isoforms, $\alpha, \beta, \delta$, and $\gamma$, each encoded by a distinct gene. Recent studies from our lab have delineated a central role for overactive CaMKII $\gamma$ in hepatocytes in the development of obesity-induced insulin resistance and diabetes (13-15). Interestingly, the same isoform is expressed by macrophages, and previous in vitro work from our lab suggested that CaMKII $\gamma$ could participate in certain types of apoptosis pathways in macrophages (16). In view of these findings, and with an eye toward identifying common upstream 
A
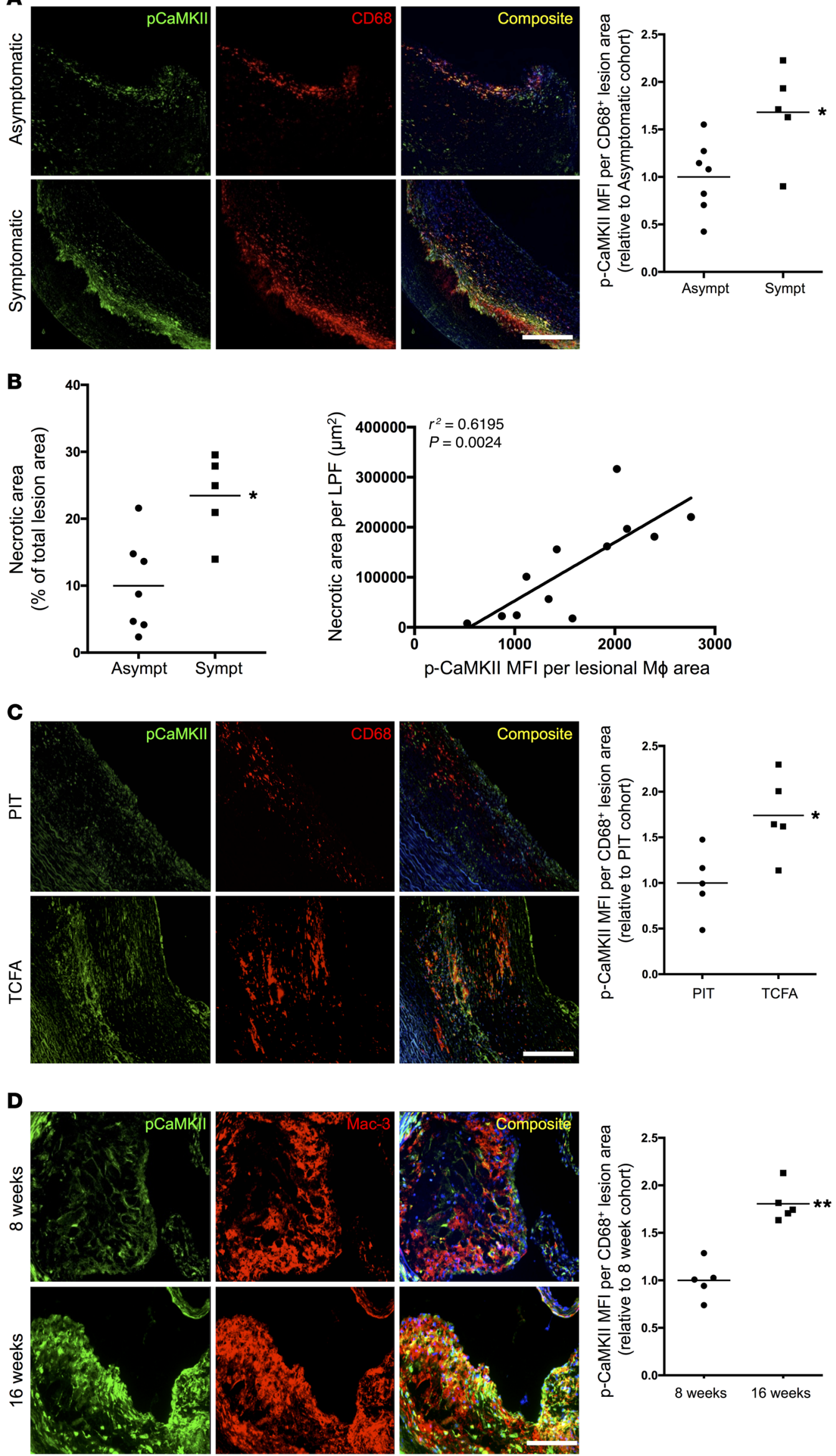

Figure 1. Expression of p-CaMKII is increased in advanced and symptomatic atherosclerotic lesions of humans and mice. (A) Human carotid specimens were obtained at time of endarterectomy from asymptomatic (Asympt) or symptomatic (Sympt) subjects with recent TIA/stroke. Fixed sections were costained for p-CaMKII (green), CD68 (red), and DAPI (nuclei; blue). Sections stained with isotype control antibodies for p-CaMKII and CD68 showed absence of signal. p-CaMKII staining was quantified as $\mathrm{MFI}$ within $\mathrm{CD}^{+} 8^{+}$cells. Data are presented relative to the average value obtained for the asymptomatic subjects' specimens ( $n=7$ asymptomatic and 5 symptomatic subjects, 2 slides per patient). Scale bar: $500 \mu \mathrm{m}$. (B) Left: adjacent sections from the human carotid endarterectomy specimens in panel $\mathbf{A}$ were stained with $\mathrm{H} \& \mathrm{E}$, and necrotic area was quantitated. Right: relationship between necrotic area and $\mathrm{p}$-CaMKII fluorescence intensity in macrophages per low power field (LPF) was plotted $\left(r^{2}=0.6195, P=0.0024\right)$. Correlation coefficient $\left(r^{2}\right)$ and $P$ values were calculated using Pearson product-moment correlation analysis. (C) Sections of human coronary arteries obtained from individual subjects at autopsy were classified as PIT or TCFA and stained and quantified as above. Data are presented relative to the average value obtained for the PIT specimens ( $n=5$ subjects in each group, 2 slides per patient). Scale bar: $250 \mu \mathrm{m}$. (D) Frozen aortic root sections from 8-week and 16-week WD-fed $L d l r^{-1-}$ mice were costained for $\mathrm{p}$-CaMKII (green), Mac-3 (red), and DAPI (blue). p-CaMKII staining was quantified as MFI within Mac $-3^{+}$cells. Data are presented relative to the average value obtained for the 8 -week specimens ( $n=5$ mice in each group, 2 slides per mouse). Scale bar: $150 \mu \mathrm{m} .{ }^{*} P<0.05,{ }^{* *} P<0.01$, Mann-Whitney $U$ test. 
pathogenic signaling pathways in different cell types that contribute to cardiometabolic disease, we sought to understand the role of macrophage CaMKII $\gamma$ in advanced atherosclerosis.

In this report, we show that macrophage CaMKII $\gamma$ is activated in advanced and symptomatic human carotid atherosclerotic lesions versus those that are less advanced or asymptomatic as well as in advanced versus early aortic root lesions in Western diet-fed (WD-fed) LDL receptor-deficient $\left(\mathrm{Ldlr}^{-/}\right)$mice. Moreover, when myeloid CaMKII $\gamma$ is deleted in WD-fed $\mathrm{Ldlr}^{-1-}$ mice, the formation of necrotic lesions is suppressed. Studies with cultured macrophages suggest that the mechanism involves a pathway through which CaMKII $\gamma$ in macrophages impairs efferocytosis by suppressing an ATF6/liver X receptor- $\alpha$ (LXR $\alpha) /$ MerTK pathway. These findings reveal an inverse link between CaMKII $\gamma$ and $\mathrm{LXR} \alpha /$ MerTK in macrophages that is relevant to necrotic plaque formation in advanced atherosclerosis and involves a new means of transcriptional regulation of LXR $\alpha$ by ATF6.

\section{Results}

CaMKII activation is increased in symptomatic and advanced human atherosclerotic disease and in advanced mouse atherosclerosis. Based on previous studies with cultured macrophages $(16,17)$, we hypothesized that CaMKII would be activated in macrophages in advanced atherosclerotic lesions and that this process would further promote the development of advanced atherosclerotic plaque characteristics. To begin to explore this hypothesis, we sought evidence of CaMKII activation in macrophages of human advanced atherosclerotic lesions. Because the major indicator of CaMKII activation is autophosphorylation of Thr287 (18), we compared immunoreactive phospho-Thr287-CaMKII (p-CaMKII) in sections of human plaques that differed in terms of clinical or pathologic progression. First, we compared sections of carotid plaques obtained from endarterectomy of patients who were either asymptomatic or had recently suffered a transient ischemic attack (TIA) or stroke. We found that most of the p-CaMKII signal colocalized with macrophages and that this signal was higher in plaques from symptomatic versus asymptomatic subjects (Figure $1 \mathrm{~A})$. Note that the mean fluorescence intensity (MFI) of p-CaMKII was determined per CD68-positive lesion area to correct for the potentially higher number of macrophages in the more advanced lesions. Importantly, the symptomatic lesions were more necrotic, and when all lesions were considered, there was a strong positive correlation between p-CaMKII and the necrotic area (Figure 1B). In contrast, there was no significant correlation between total CaMKII $\gamma$ signal in lesional macrophages and the necrotic area (Supplemental Figure 1A; supplemental material available online with this article; https://doi.org/10.1172/JCI94735DS1). Furthermore, there was a significant relationship between necrotic area and p-CaMKII MFI per total lesion area (Supplemental Figure $1 B)$. Given the role of CaMKII in smooth muscle cells $(11,19)$, we compared p-CaMKII MFI associated with smooth muscle $\alpha$-actinpositive (SM $\alpha \mathrm{A}$-positive) cells in lesions and found no significant differences between the plaques of asymptomatic and symptomatic patients (Supplemental Figure 1C). Thus, although CaMKII is active in both macrophages and smooth muscle cells in atherosclerosis, it is primarily the activity in macrophages that increases in symptomatic lesions. Finally, in a separate cohort of patients, we assayed lesional p-CaMKII using sections of coronary artery plaque classified as either pathological intimal thickening (PIT), which is a relatively stable type of lesion, or TCFA, which is an advanced type of lesion. The p-CaMKII signal per CD68-positive lesion area was higher in the TCFA lesions versus PIT lesional macrophages (Figure 1C).

In anticipation of the molecular-genetic causation studies in mice that follow, we assayed $\mathrm{p}$-CaMKII in atherosclerotic lesions from $\mathrm{Ldlr}^{-/}$mice fed a WD for 8 weeks, which resulted in relatively early lesions, and in mice fed the WD for 16 weeks, which led to advanced plaques. As with human lesions, p-CaMKII colocalized mostly with lesional macrophages, and the p-CaMKII signal was more intense in the advanced versus early plaques (Figure 1D; isotype controls are shown in Supplemental Figure 1D). No difference was found in total CaMKII $\gamma$ fluorescence in the macrophage-rich areas of 8-week versus 16-week groups, indicating a true increase in p-CaMKII/total CaMKII ratio (Supplemental Figure 1E).

Genetic targeting of myeloid CaMKII $\gamma$ in WD-fed $\mathrm{Ldlr}^{-}$mice leads to smaller necrotic core area and improved efferocytosis in atherosclerotic plaques. Given the increase in p-CaMKII in advanced human and mouse lesions, and because macrophages exclusively express the $\gamma$ isoform of CaMKII $(16,17)$, we hypothesized that the loss of CaMKII $\gamma$ in myeloid cells would suppress development of advanced atherosclerotic plaque characteristics. To test this hypothesis, we bred Camk2 $g^{f / / l}$ mice onto the $\mathrm{Ldlr}^{-/}$background and then further crossed these mice to lysozyme M-Cre (LysM-Cre) mice in order to drive myeloid-lineage-specific deletion of the gene. The resulting Camk2 $g^{f l / f l} \mathrm{Ldlr}^{-/} \mathrm{LysM}-\mathrm{Cr}^{+/-}$mice (M-CaMKII-KO mice) and littermate control Camk2 $g^{f l / f l} \mathrm{Ldlr}^{-/}$ mice (control mice) were fed the WD for 12 weeks. There were no significant differences in body weight, insulin, glucose, plasma cholesterol, plasma lipoprotein distribution of cholesterol, or plasma triglycerides between the 2 groups of mice (Supplemental Figure 2, A-F). Additionally, we found no differences in circulating total white blood cell, monocyte, neutrophil, or platelet numbers (Supplemental Figure 2, G-J), and there was also no difference in the percentage of lesional macrophages, total $\mathrm{CD}^{+} \mathrm{T}$ cells, or regulatory $\mathrm{T}$ cells between the 2 groups (Supplemental Figure 2, $\mathrm{K}-\mathrm{M})$. As expected from the specificity of LysM-Cre (20), M-CaMKII-KO mice showed no detectable CaMKII $\gamma$ signal in macrophage-rich areas of aortic root lesions (Supplemental Figure 1E), but the signal in lesional $\mathrm{SM} \alpha \mathrm{A}^{+}$cells and $\mathrm{vWF}^{+}$endothelial cells was retained (Supplemental Figure 1F).

Our primary end points were 2 features associated with the clinical progression of human plaques: necrotic core area and thinning of the collagenous fibrous cap that covers and "stabilizes" advanced lesions (21). Inspection of aortic root plaques suggested a significant decrease in necrotic core area in the M-CaMKIIKO lesions compared with control lesions, and this was verified by quantitative image analysis (Figure 2A). Importantly, overall lesion area (Figure 2B) was similar between the 2 cohorts, indicating a true decrease in the necrotic core/lesion area ratio (Figure $2 \mathrm{C})$. A decrease in this ratio is often seen when a process specific to advanced plaque progression has been targeted $(21,22)$. We also found a substantial increase in fibrous cap thickness in the M-CaMKII-KO lesions (Figure 2D), which is another indicator of plaque stabilization. Consistent with this interpretation, we found 
A
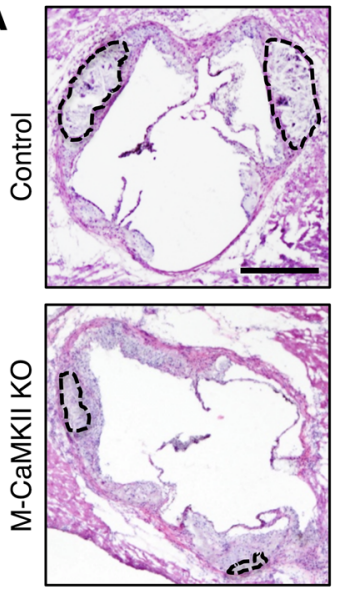

D
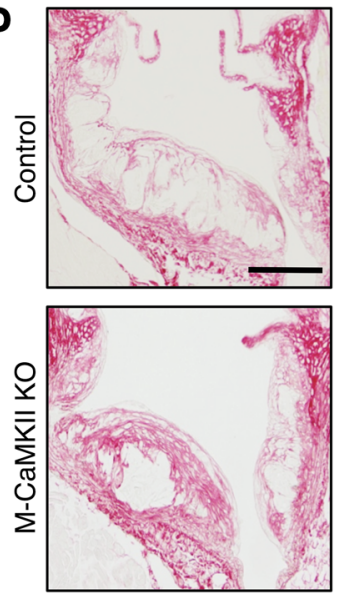
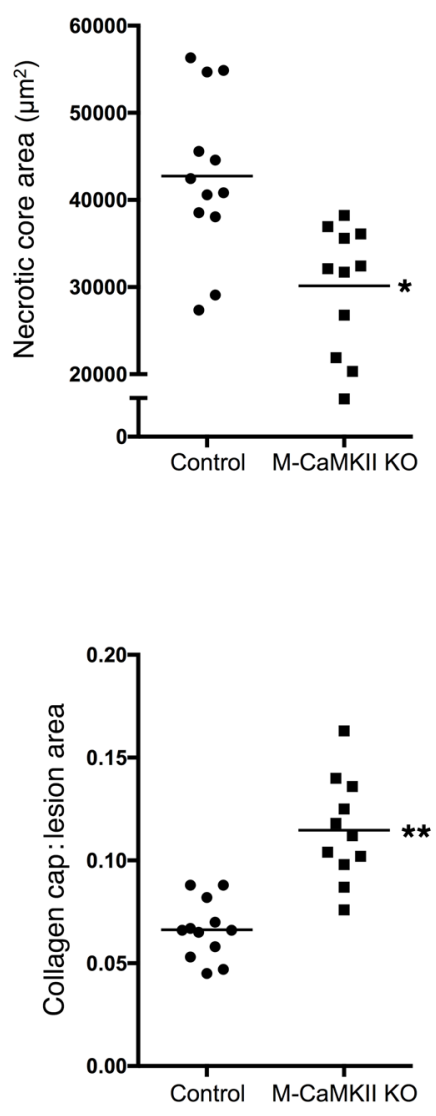

$\mathbf{E}$
B

C
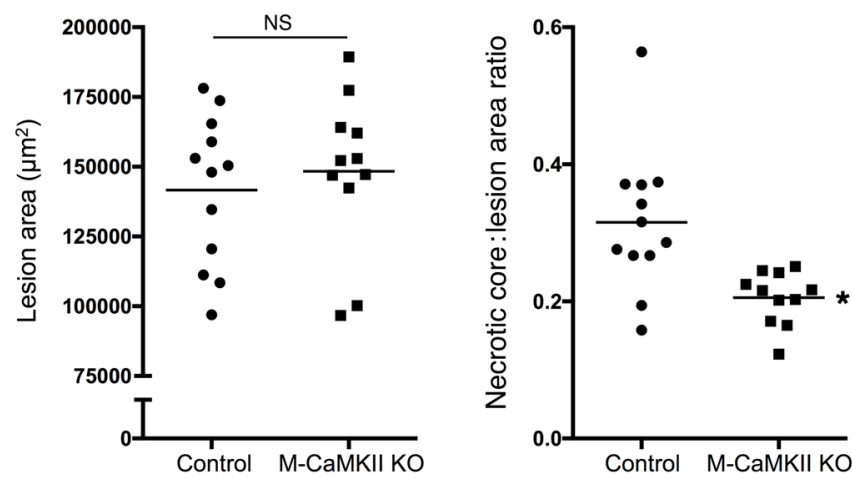

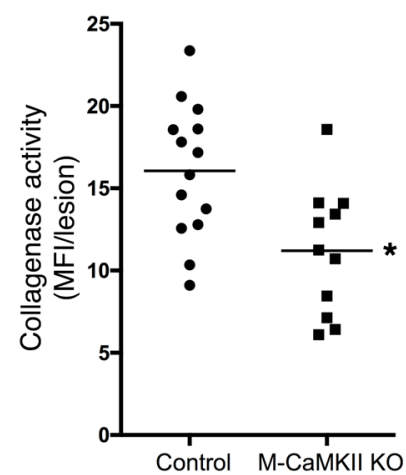

Figure 2. The lesions of WD-fed M-CaMKII-KO LdIr ${ }^{-1-}$ mice have less plaque necrosis and thicker fibrous caps than control LdIr ${ }^{-1-}$ mice. Mice were fed WD for 12 weeks, and paraffin-embedded sections of the aortic root were stained with $\mathrm{H} \& \mathrm{E}$. (A) Cross sections were quantified for necrotic core area. Left: representative images with necrotic cores outlined in black. Scale bar: $200 \mu \mathrm{m}$. Right: quantitation of necrotic core areas ( $n=12$ control and 11 M-CaMKII-KO mice). (B) Cross sections were quantified for total lesion area based on the H\&E sections in $\mathbf{A}$. (C) Based on the results shown in $\mathbf{A}$ and $\mathbf{B}$, necrotic core/lesion area ratio was calculated. (D) Aortic root sections were stained with picrosirius red. For each section, cap thickness was measured at the lesional midpoint and both shoulder regions and then averaged and quantified as ratio of collagen cap thickness to lesion area ( $n=12$ control and $n=11 \mathrm{M}$-CaMKII-KO mice). Scale bar: $100 \mu \mathrm{m}$. (E) Collagenase activity was quantified by fluorescence microscopy in frozen aortic root sections from a separate cohort of mice fed the WD for 12 weeks; note that the $\mathrm{KO}$ group in this cohort showed a similar reduction in plaque necrosis and a similar increase in fibrous cap thickness versus the WT control group, as in the previous experiment. ( $n=14$ control and $n=11 \mathrm{M}$-CaMKII-KO mice). ${ }^{*} P<0.01$; ${ }^{* *} P<0.001$, unpaired $t$ test.

that M-CaMKII-KO lesions had less collagenase activity (Figure 2E), which might contribute to the thicker collagen caps in the lesions from these mice $(23,24)$. A similar decrease in necrotic core/lesion area was seen in brachiocephalic arterial lesions of $\mathrm{M}-\mathrm{CaMKII}-\mathrm{KO}$ versus control mice (Supplemental Figure 3, A-C).

We then sought to further characterize important features of advanced atherosclerotic disease in these mice. Elevated ER stress, particularly increased expression of mediators of the unfolded protein response (UPR), is another feature of advanced human and mouse plaques $(22,25,26)$. Using laser capture microdissection (LCM) of aortic root sections followed by quantitative real-time quantitative PCR (RT-qPCR), we found significantly less expression of mRNAs encoding 3 genes that are induced by the UPR: Ddit3 (encoding CHOP), spliced Xbp1, and Dnajb9 (encoding ERdj4) (Supplemental Figure 4, A-C). Because advanced lesions are characterized by the presence of proinflammatory cytokines, we also determined the levels of $I l 1 b, I l 6$, and
Tnfa mRNA in LCM samples of aortic root lesions and found significantly lower levels of all 3 cytokine mRNAs in the lesions of M-CaMKII-KO mice (Supplemental Figure 4, D-F). To gain insight into whether these differences are cell intrinsic due to the absence of CaMKII $\gamma$ in macrophages, we examined the response of control and CaMKII-KO bone marrow-derived macrophages to low-dose LPS. There was a marked reduction in the expression of Il6 in CaMKII-KO macrophages and a smaller, though significant, reduction in Il1b, but Tnfa was not reduced (Supplemental Figure 4, G-I). Thus, the decrease in inflammation in M-CaMKII-KO lesions may be caused by the direct effect of CaMKII $\gamma$ deficiency on inflammatory signaling in macrophages as well as by secondary causes, such as plaque necrosis. Taken together, these findings demonstrate that genetic targeting of myeloid CaMKII $\gamma$ protects WD-fed $\mathrm{Ldlr}^{-1-}$ mice from developing several key features of a type of advanced plaque that can precipitate acute vascular events in humans. 

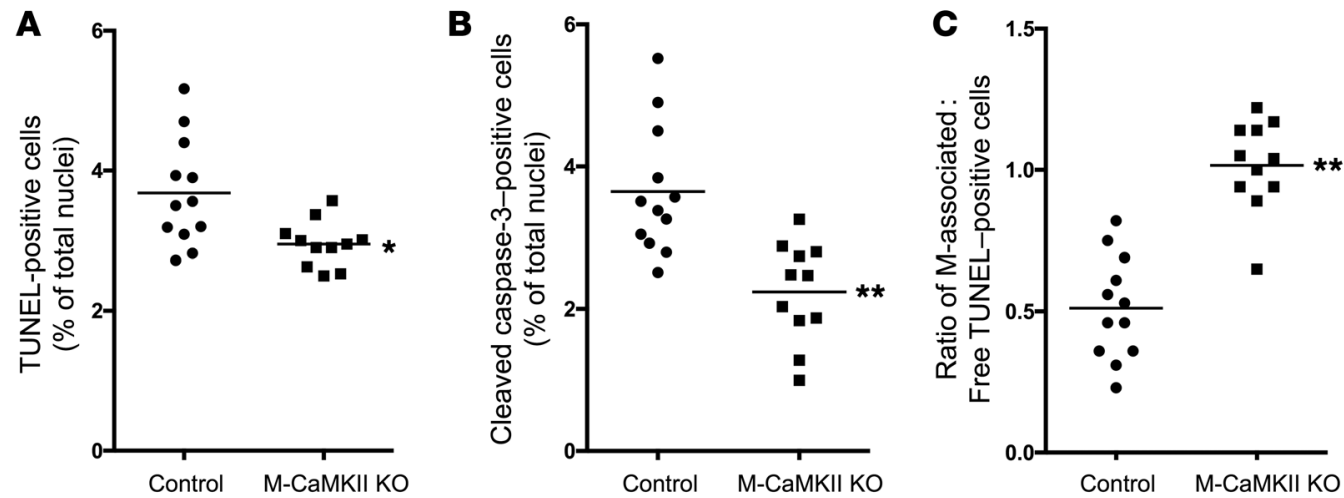

Figure 3. The lesions of WD-fed M-CaMKII-KO LdIr/- mice have less apoptosis and improved efferocytosis. Aortic root lesions from the mice described in Figure 2 were assayed as follows: (A) aortic root sections were stained for TUNEL and DAPI and quantified as the percentage of TUNEL-positive cells of the total nuclei. (B) Aortic root sections were stained with an antibody specific for cleaved caspase-3 and DAPI. Data are presented as the percentage of positive cells of total nuclei. (C) Aortic root sections were costained for Mac-3 and TUNEL. Each TUNEL-positive cell was determined to be either associated with a macrophage or not associated with a macrophage, and the data are presented as the ratio of macrophage-associated apoptotic cells to free apoptotic cells. For all panels, $n=12$ control and $n=11 \mathrm{M}$-CaMKII-KO mice. ${ }^{*} P<0.01$; ${ }^{* *} P<0.001$, unpaired $t$ test.

A major cause of necrotic core formation in advanced atherosclerosis is defective phagocytic clearance of apoptotic cells (efferocytosis) within lesions, leading to secondary necrosis of the uncleared cells and necrotic cell-induced inflammation (24). We first assayed apoptotic cells in lesions by measuring the percentage of lesional cells that showed either TUNEL positivity or caspase-3 activation. By either measurement, the lesions of M-CaMKII-KO mice had fewer apoptotic cells compared with control mice (Figure 3, A and B). To determine whether this finding was associated with defective efferocytosis, we used a standard assay in which lesions costained for TUNEL and macrophages (Mac-3) are quantified for $\mathrm{TUNEL}^{+}$cells that are either associated with a macrophage, indicative of an efferocytic event, or not associated with macrophages (free) (10). Data are presented as the ratio of associated/free $\mathrm{TUNEL}^{+}$cells, with higher values representing higher levels of efferocytosis. We found a marked increase in this measure of efferocytosis in the M-CaMKII-KO lesions compared with lesions from the control group (Figure $3 \mathrm{C}$ ). These data suggest that at least one mechanism for the decreased number of apoptotic cells in the lesions of M-CaMKII-KO mice is improved efferocytosis. Most importantly, improved efferocytosis could also explain the decrease in plaque necrosis in M-CaMKII-KO mice, i.e., by preventing secondary necrosis of lesional apoptotic cells.

CaMKII-KO macrophages and lesions express higher levels of the efferocytosis receptor MerTK. To further elucidate the role of macrophage CaMKII $\gamma$ in efferocytosis, we assayed efferocytosis in vitro using bone marrow-derived macrophages from control and CaMKII-KO mice. Consistent with the lesional data, CaMKII-KO macrophages displayed increased efferocytosis compared with control macrophages (Figure 4A). Because efferocytosis is a 2-step process in which macrophages first bind apoptotic cells via specialized efferocytosis receptors and then internalize the apoptotic cells, we repeated this assay at $4^{\circ} \mathrm{C}$, which allows for binding of apoptotic cells, but not their ATP-dependent internalization. Under these conditions, CaMKII-KO macrophages showed enhanced apoptotic cell binding (Figure
4B), suggesting improved apoptotic cell recognition. We then compared CaMKII-KO and control macrophages for a number of efferocytosis receptors proposed to have a role in atherosclerosis. We found no differences in the expression of $\beta_{5}$ integrin, CD36, CD61, CD206, CD300F, or Tim4. CaMKII-KO macrophages did, however, have a marked enhancement in cell-surface expression of MerTK (Figure 4C), which plays an important role in efferocytosis in advanced atherosclerotic lesions (9, 10, 27). CaMKII-KO macrophages also had an increase in Mertk mRNA (Figure 4D). To confirm our findings, we used the CaMKII inhibitor KN93 (16) and showed that this compound, but not the inactive homolog KN92, induced an increase in Mertk mRNA, cell-surface MerTK, and efferocytosis (Supplemental Figure 5, A-C). Moreover, KN93 was unable to induce further increases in these parameters in CaMKII-KO macrophages, indicating an on-target effect of the inhibitor. Finally, we examined control and M-CaMKII-KO atherosclerotic lesions for MerTK expression by immunofluorescence microscopy and found that M-CaMKII-KO lesions expressed significantly more of the protein than control lesions (Figure 4E). Thus, the increase in efferocytosis in CaMKII-KO macrophages in vitro and in atherosclerosis is associated with an increase in MerTK, which is a known mediator of macrophage efferocytosis in atherosclerosis.

CaMKII deficiency in macrophages increases the expression of LXRa, which induces MerTK. To explain the link between CaMKII deficiency and increased MerTK-mediated efferocytosis, we reasoned that CaMKII $\gamma$ deficiency in macrophages might lead to an increase in a Mertk inducer. In this context, we assayed an Mertk inducer that is relevant to atherosclerosis, LXR (28), and found that Nr1h3 mRNA (encoding LXR $\alpha$ ), LXR $\alpha$ protein, and one of LXR's gene targets, Abcal, were indeed increased in CaMKII-KO macrophages (Figure 5, A and B). Interestingly, Nr1h2 mRNA (encoding LXR $\beta$ ) was not increased (Figure 5A). Confirming these findings, KN93, but not KN92, induced an increase in Nr1h3 and Abca1 mRNA without any change in Nr1h2 levels (Supplemental Figure 5, D-F). To test causation with regard to Mertk induction, we treated 
A

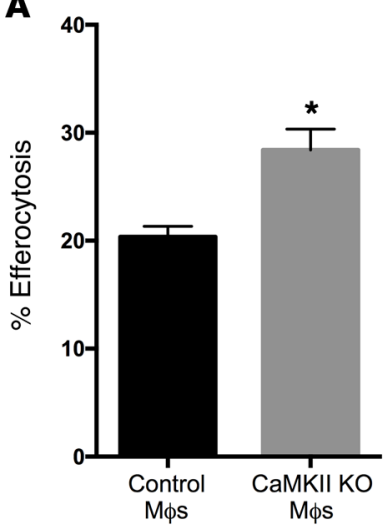

D

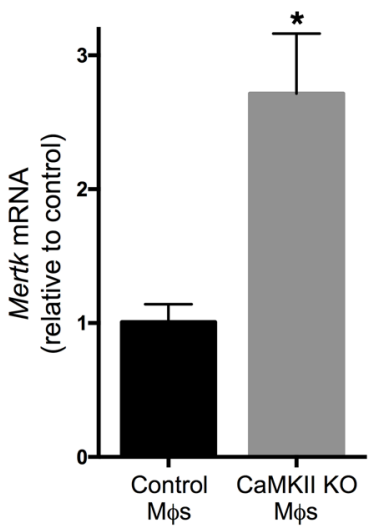

B

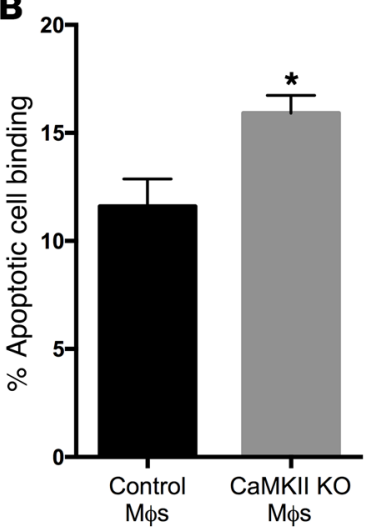

$\mathbf{E}$

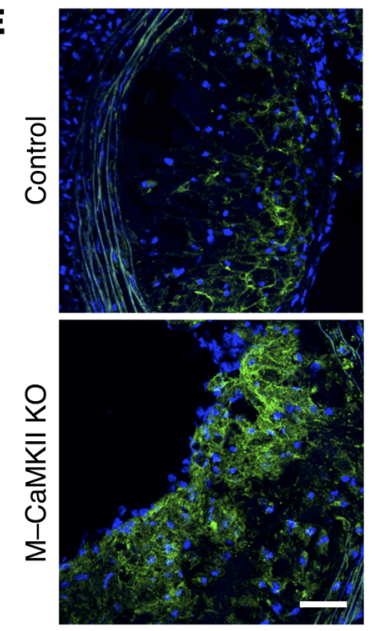

C
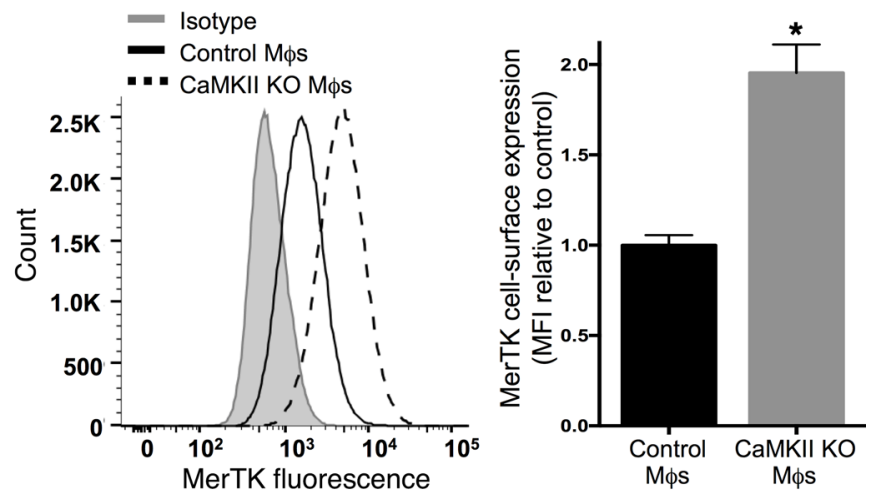

Figure 4. Macrophages from M-CaMKII-KO mice show improved efferocytosis and increased expression of the efferocytosis receptor MerTK both in vitro and in atherosclerotic lesions. Bone marrow-derived macrophages (M $\phi \mathrm{s}$ ) from control or M-CaMKII-KO mice were incubated for 45 minutes with PKH67-green linker-labeled apoptotic Jurkat cells at a 3:1 apoptotic cell/macrophage ratio. One set of wells was incubated at $37^{\circ} \mathrm{C}$ to assess efferocytosis (A) and the other at $4^{\circ} \mathrm{C}$ to assess apoptotic cell binding (B). Results are shown as mean $+\mathrm{SEM}, n=3$ experiments; ${ }^{*} P<0.001$ by unpaired $t$ test. (C) Control and CaMKII-KO macrophages were immunostained for MerTK and then subjected to flow cytometry. Left: representative flow cytometric histogram. Right: quantification of MerTK MFI. Data are normalized to the average value from control macrophages and presented as fold change. (D) mRNA was harvested from control and CaMKII-KO macrophages and quantified by RT-qPCR for Mertk, with normalization to RplpO (36B4) mRNA. Data are presented as relative to the control macrophage value. Results are shown as mean + SEM. $n=3$ experiments. ${ }^{*} P<0.001$, unpaired $t$ test (A-D). (E) Aortic root sections from the 2 cohorts of mice described in Figure 2 were immunostained for MerTK and DAPI (nuclei) and analyzed by confocal microscopy. Scale bar: 50 m. Images were quantified for MerTK MFI per lesion area. $n=5$ mice in each group. ${ }^{* *} P<0.05$, Mann-Whitney $U$ test.

macrophages from control and CaMKII-KO mice with siRNA targeting LXR $\alpha$. As above, CaMKII-KO macrophages expressed higher levels of Mertk mRNA than control macrophages, and in response to $\operatorname{siLXR} \alpha$, this increment was abrogated (Figure 5C). Similar results were found for Abca1 mRNA, cell-surface MerTK, and efferocytosis (Figure 5, C-E). We next examined the potential in vivo relevance of our findings by using LCM to assess relevant mRNAs in the atherosclerotic lesions of control and M-CaMKII-KO mice. Consistent with our in vitro findings, lesions from M-CaMKII-KO mice contained significantly higher levels of $N r 1 h 3$ (LXR $\alpha)$ and Abca1 mRNAs than did lesions from control mice, while there was no significant difference in the level of Nr1h2 (LXR $\beta$ ) mRNA (Figure 5F). These data provide a plausible mechanism linking macrophage CaMKII $\gamma$ deficiency to increased MerTK-mediated efferocytosis, namely, through an LXR $\alpha /$ MerTK induction pathway.
CaMKII deficiency in macrophages disrupts an HDAC4/DACH1/ ATF6 pathway that regulates LXR and MerTK. Previous studies from our lab have elucidated a pathway in hepatocytes in which activated CaMKII phosphorylates HDAC4, causing its nuclear exclusion (14). Cytoplasmic HDAC4 is unable to SUMOylate and target the nuclear corepressor DACH1 for degradation. The resulting increase in nuclear DACH1 represses expression of the transcription factor ATF6, leading to decreased expression of ATF6 gene targets (14).

We therefore sought to determine whether this pathway might also be present in macrophages and possibly relevant to the increase in LXR $\alpha$ in CaMKII $\gamma$-deficient macrophages. As was the case with hepatocytes, pHDAC4 and DACH1 were lower and ATF6 was higher in CaMKII-KO versus control macrophages (Figure 6A). Note that the increase in ATF6 was apparent at both the protein level (Figure 6A, showing cleaved/nuclear form) and the mRNA level (Figure 6B). Next, we wondered whether $\mathrm{Nr} 1 \mathrm{~h} 3$ 
A

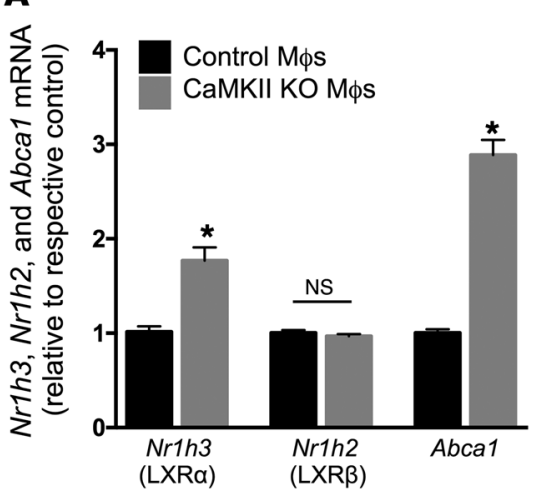

B

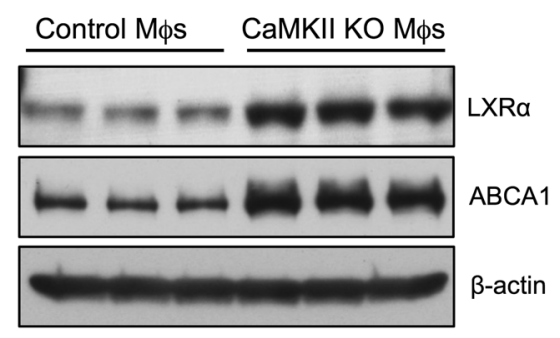

C

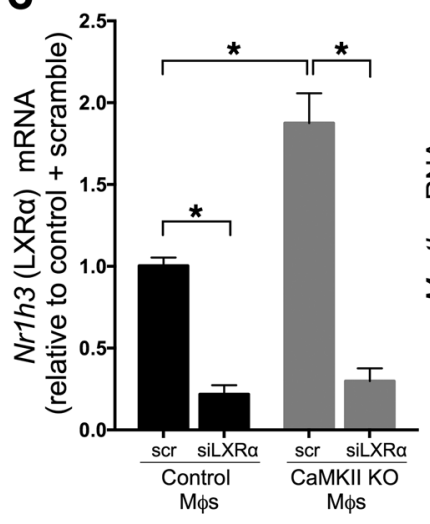

D

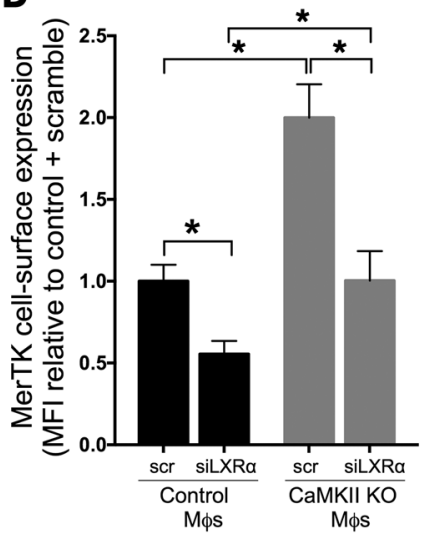

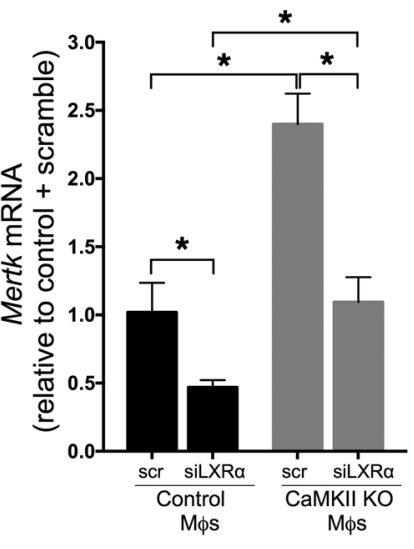

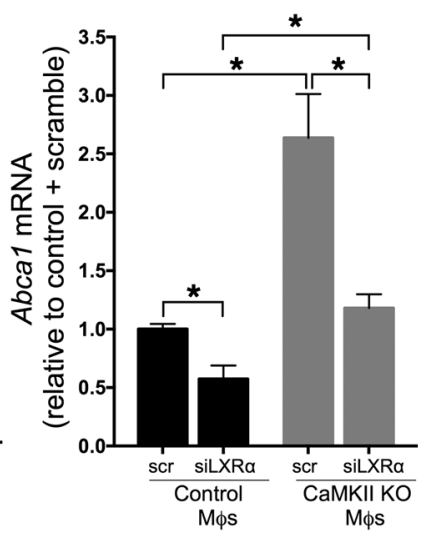

E

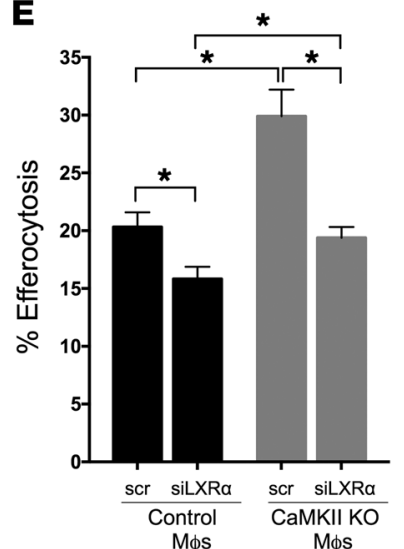

Figure 5. CaMKII deficiency in macrophages increases the expression of LXRa, which induces MerTK. (A) mRNA was harvested from control and CaMKII-KO macrophages and quantified by RT-qPCR analysis for Nr1h3 (LXR $\alpha)$, Nr1h2 (LXRß), and Abca1 and normalized to RplpO (36B4). Data are shown as relative to the value for control macrophages. Results are shown as mean + SEM. $n=3$ experiments. ${ }^{*} P<0.001$, unpaired $t$ test. (B) Lysates of control and CaMKII-KO macrophages were immunoblotted for $\operatorname{LXR} \alpha, A B C A 1$, and $\beta$-actin. $n=3$ experiments. (C) Macrophages were transfected with scrambled RNA (scr) or siRNA targeting LXR $\alpha(\operatorname{siLXR} \alpha)$ and, after 48 hours, assayed for Nr1h3, Nr1h2, and Abca1 mRNA. Data are shown as relative to the value for control macrophages. Results are shown as mean + SEM. $n=3$ experiments. ${ }^{*} P<0.001,2$-way ANOVA with post hoc Tukey's analysis. (D) Macrophages were labeled with MerTK antibody at $4^{\circ} \mathrm{C}$ and subjected to flow cytometric analysis. MFI data are shown as relative to the value for control macrophages. Results are shown as mean + SEM. $n=3$ experiments. ${ }^{*} P<0.001,2$-way ANOVA with post hoc Tukey's analysis. (E) Macrophages were incubated for 45 minutes with a 3:1 ratio of PKH67-green linker-labeled apoptotic Jurkat cells to macrophages and assayed for efferocytosis by image analysis of fluorescent microscopy images. Results are shown as mean + SEM. $n=3$ experiments.

${ }^{*} P<0.001,2$-way ANOVA with post hoc Tukey's analysis. (F) RNA was obtained from aortic root sections by LCM. Nrih3, Nr1h2, and Abca1 mRNA was quantified by RT-qPCR with normalization to Gapdh. Data are shown as relative to the value for lesional mRNA from the control cohort. $n=5$ in each group. ${ }^{*} P<0.05$, Mann-Whitney $U$ test.
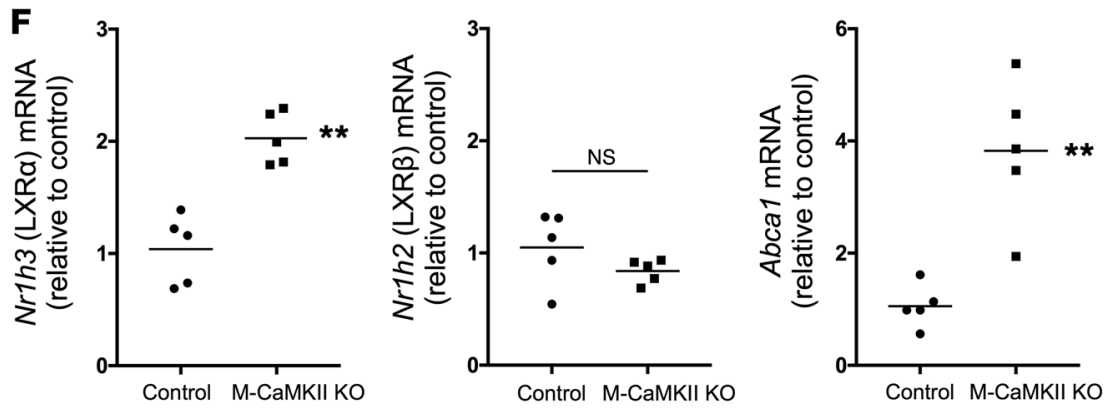

$(\mathrm{LXR} \alpha)$ might be a transcriptional target of ATF6. Indeed, we found that the Nr1h3 (LXR $\alpha)$ gene, but not the Nr1h2 (LXR $\beta)$ gene, has a consensus ATF6-binding site in intron 1 that is conserved across species, and ChIP analysis showed interaction of ATF6 with this site (Figure 6C). Moreover, according to the ENCODE ChIP-seq database, this site is surrounded by strong signals for POLR2A and $\mathrm{H} 3 \mathrm{~K} 4 \mathrm{~m} 3$, suggesting that it is an active transcriptional regulatory region (29-31). To determine whether ATF6 might regulate LXR expression, we silenced ATF6 using siRNA and found significant decreases in Nr1h3 (LXR $\alpha$ ) mRNA and its target Abca1 (Figure 6D). 


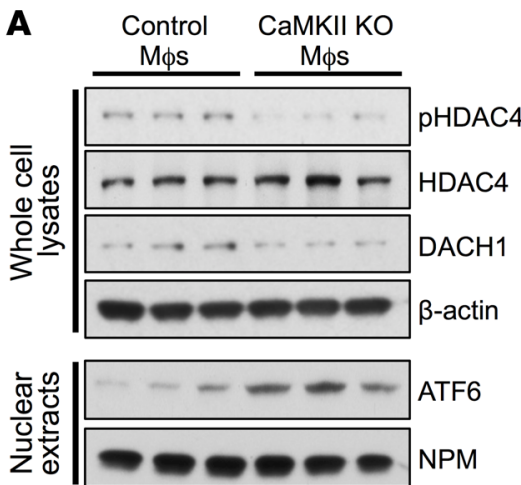

C

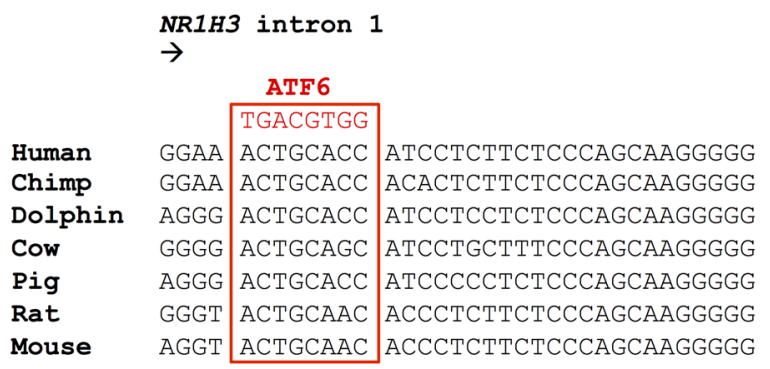

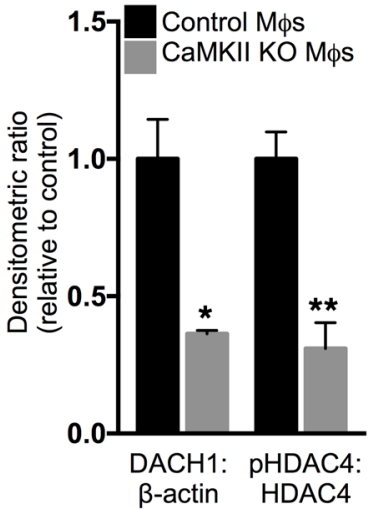

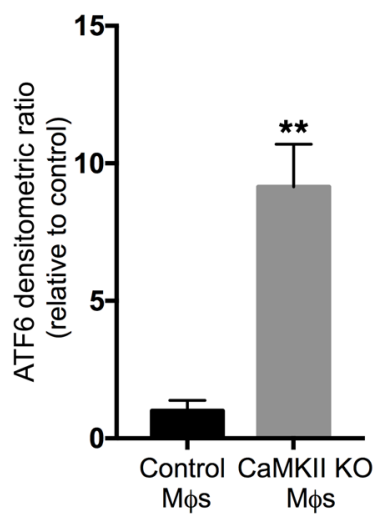

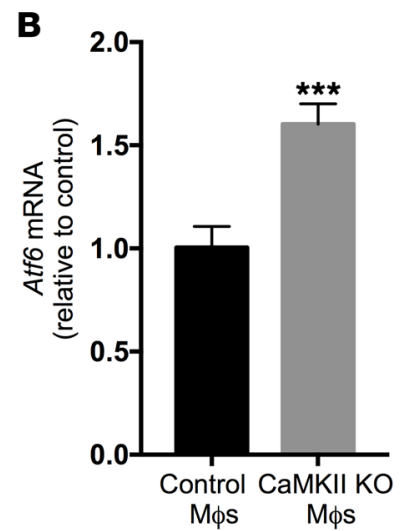
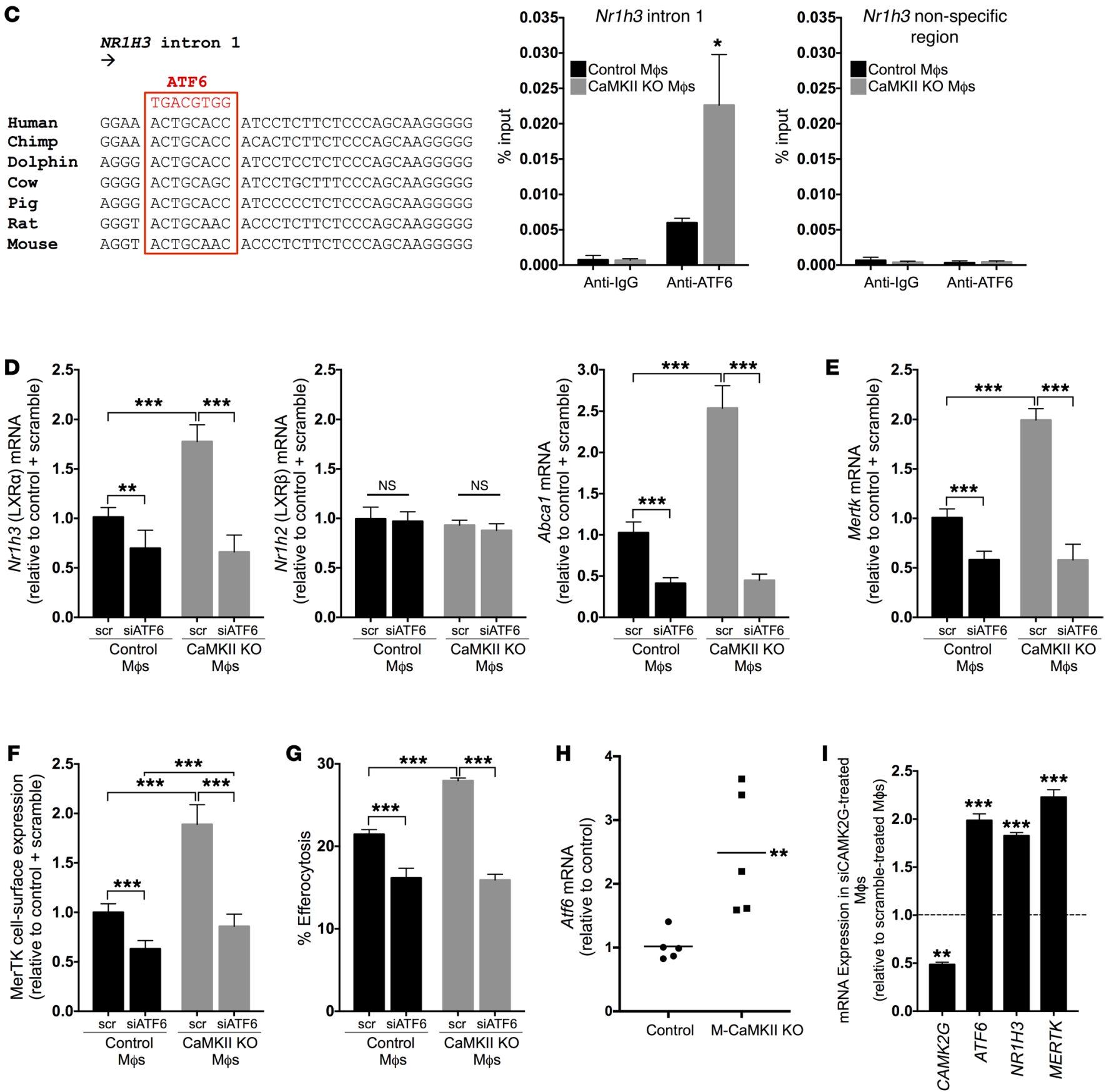
Figure 6. CaMKII deficiency in macrophages disrupts an HDAC4/DACH1/ ATF6 pathway that regulates LXR $\alpha$ and MerTK. (A) Whole cell or nuclear extracts from control and CaMKII-KO macrophages were immunoblotted using antibodies against p-HDAC4 (pHDAC4), HDAC4, DACH1, $\beta$-actin, ATF6, and nucleophosmin (NPM). Left: representative immunoblot. Right: densitometry. ${ }^{*} P<0.05$; ${ }^{*} P<0.01$, unpaired $t$ test. (B) Macrophage mRNA was quantified by RT-qPCR for Atf6. Data are presented as relative to the value for control macrophages. ${ }^{* *} P<0.001$, unpaired $t$ test. (C) Left: conserved ATF6 consensus sequence in intron 1 of the $N r 1 h 3$ $(\mathrm{LXR} \alpha)$ gene. Right: macrophages from control and CaMKII-KO mice were subjected to ChIP analysis using anti-ATF6 or IgG control. The intronic region containing the ATF6-binding site and a nonconsensus sequence were amplified by RT-qPCR and normalized to input DNA. $n=4$ biologic replicates. ${ }^{*} P<0.05$, unpaired $t$ test. (D) Macrophages were transfected with siRNA targeting ATF6 (siATF6) and analyzed for the indicated mRNAs as in Figure 5C. ${ }^{* *} P<0.01$; ${ }^{* *} P<0.001,2$-way ANOVA with post hoc Tukey's analysis. (E-C) Macrophages were transfected with siATF6 as above and analyzed for Mertk mRNA, cell-surface MerTK by flow cytometry, and efferocytosis. ${ }^{* *} P<0.001$, 2-way ANOVA with post hoc Tukey's analysis. (H) RNA from aortic root sections was quantified for Atf6 mRNA as in Figure 5F. $n=5$ mice per group. ${ }^{* *} P<0.01$, Mann-Whitney $U$ test. (I) Human monocyte-derived macrophages were transfected with scrambled RNA or siRNA targeting CAMK2G and harvested 72 hours later for analysis of CAMK2G, ATFG, NR1H3 (LXR $\alpha$ ), and MERTK. Dotted line represents the average value obtained with scramble RNA for each gene of interest. ${ }^{* *} P<0.01 ;{ }^{* *} P<0.001$, unpaired $t$ test. For all experiments, results are shown as mean + SEM. $n=3$ experiments unless noted otherwise.

No significant change in Nr1h2 (LXR) was observed. Consistent with a link between LXR $\alpha$ and MerTK, silencing of ATF6 lowered Mertk mRNA, cell-surface expression of MerTK, and efferocytosis in both control and, most notably, in CaMKII-KO macrophages (Figure 6, E-G). Furthermore, we found significantly higher levels of Atf6 mRNA in the lesions of M-CaMKII-KO mice versus control mice (Figure 6H). Finally, the key findings were reproduced in human monocyte-derived macrophages: approximately $50 \%$ silencing of CAMK2G mRNA using siRNA led to significant increases in ATF6, NR1H3 (LXR $\alpha)$, and MERTK mRNA expression (Figure 6I). These combined data show that CaMKII $\gamma$ deficiency in macrophages enhances MerTK-mediated efferocytosis through a new pathway in which ATF6 induces $\operatorname{LXR} \alpha$.

Because CaMKII is activated as atherosclerosis progresses, we tested whether overexpression of an active form of CaMKII in cultured, nonstimulated macrophages was sufficient to suppress ATF6, LXR $\alpha$, and efferocytosis. For this purpose, we transduced bone marrow-derived macrophages with a viral vector encoding a constitutively active form of CaMKII (CA-CaMKII, which has a T287D mutation) $(15,32)$ or a control virus expressing LacZ. In macrophages transduced with CA-CaMKII, there was significantly less Atf6, Nr1h3 (LXR $\alpha)$, and Mertk mRNA (Figure 7, A-C) and decreased levels of LXR $\alpha$ and MerTK protein (Figure 7D) compared with macrophages transduced with LacZ. Further, macrophages transduced with CA-CaMKII showed a decrease in efferocytosis (Figure 7E). These data, together with both the lesional data presented earlier and the previously published studies on the role of MerTK in advanced plaques $(9,10$, 27), support the concept that activation of CaMKII $\gamma$ in lesional macrophages, by suppressing an ATF6-LXR $\alpha$-MerTK module, contributes to defective efferocytosis and plaque necrosis in advanced atherosclerotic lesions.

\section{Discussion}

The development of large necrotic cores with thin overlying collagen caps is a hallmark of a particular type of human plaque that can trigger acute thrombo-occlusive vascular events $(5,21)$. Two processes that can lead to plaque necrosis are defective efferocytosis of apoptotic cells and primary cellular necroptosis (7, 33-37). Defective efferocytosis can result if apoptotic cells resist uptake by phagocytes, as described recently for atherosclerosis (38), or if the phagocytes themselves have an impairment in apoptotic cell binding or internalization. The work here defines a process that may help explain defective apoptotic cell recognition by advanced lesional macrophages. As described, activated CaMKII in these macrophages leads to decreased expression of a key atherosclerosis-relevant efferocytosis receptor, MerTK. Interestingly, another process in advanced lesions can lead to the ADAM17-mediated proteolytic degradation of MerTK, and prevention of this process using genetic engineering to render MerTK resistant to proteolysis also suppresses plaque progression (39).

While we have accumulated substantial evidence to suggest that the ATF6/LXR $\alpha /$ MerTK/efferocytosis pathway is a major mechanism contributing to the improvement in necrotic core area in the M-CaMKII-KO mouse, it is possible that other mechanisms contributed to the overall lesional phenotype. Our laboratory previously reported that CaMKII $\gamma$ activation in macrophages can trigger apoptosis (16), and we did see a decrease in apoptotic cells in M-CaMKII-KO lesions (Figure 3A). However, based on the in situ efferocytosis data and our mechanistic work in the current study, together with the previous literature on lesional efferocytosis and MerTK $(9,10,27)$, we believe that the dominant mechanism accounting for the decrease in apoptotic cells in $\mathrm{M}$-CaMKII-KO lesions is the enhanced clearance of these cells by lesional macrophages. Nonetheless, it is possible that decreased lesional apoptosis, perhaps due to a separate effect of macrophage CaMKII as discussed below, could have contributed to the plaque phenotype in these mice.

The increase in fibrous cap thickness and lower collagenase activity in M-CaMKII-KO lesions raises the question of whether overactive macrophage CaMKII impairs inflammation resolution-induced repair processes in advanced lesions. Inflammation resolution, a process through which specific molecules restore tissue homeostasis after an inflammatory response, is impaired in advanced human and mouse plaques (40-42). Moreover, therapeutic restoration of resolution mediators can block plaque progression in mice (41-44). In this context, it is possible that the loss of macrophage CaMKII $\gamma$ improves resolution in plaques. If so, one mechanism could be related to our recent finding that CaMKII activation in macrophages preferentially channels the common precursor lipid mediator arachidonic acid into proinflammatory leukotriene $B_{4}$ rather than proresolving lipoxin $A_{4}$ (17). If the resolution process is improved in $\mathrm{M}-\mathrm{CaMKII}-\mathrm{KO}$ lesions, as we predict, this might explain the improvement in protective fibrous cap formation (45). However, it is also possible that M-CaMKIIKO directly inhibits expression or activity of matrix metalloproteinases (see Figure 2E) $(46,47)$, which have been implicated in advanced plaque progression $(48,49)$. Another possible link between inflammation resolution and the findings reported herein is that efferocytosis in general, and MerTK signaling in par- 

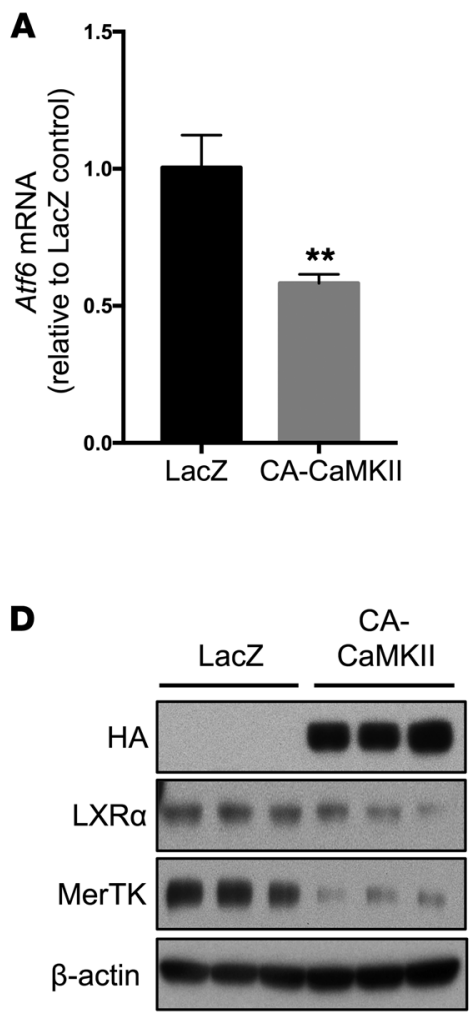

B
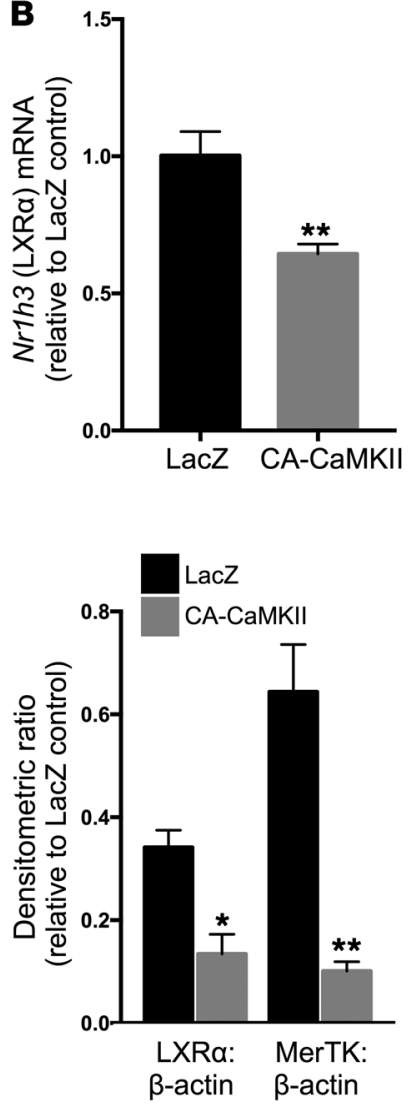

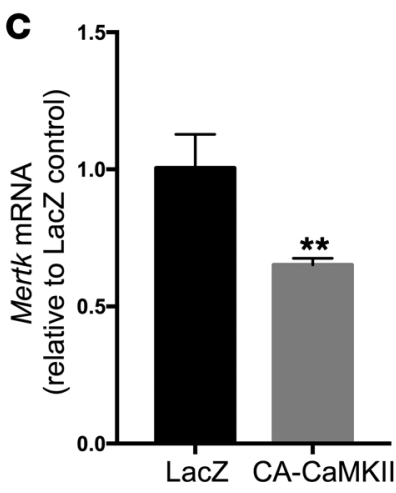

$\mathbf{E}$

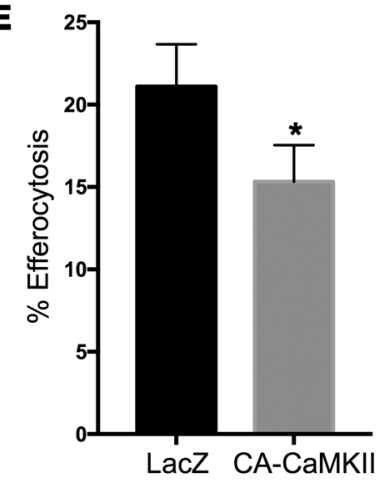

Figure 7. Constitutively active CaMKII in macrophages reduces ATF6, LXR $\alpha$, MerTK, and efferocytosis. Bone marrow-derived macrophages were transduced with $200 \mathrm{MOI}$ of adenovirus encoding an HA-tagged constitutively active CaMKII (HA CA-CaMKII) or LacZ (control) and harvested after 60 hours. (A-C) mRNA was harvested and quantified by RT-qPCR for Atf6, Nr1h3 (LXR $\alpha$ ), and Mertk. Data are presented as relative to the LacZ value. Data are shown as mean + SEM. $n=3$ experiments. ${ }^{*} P<0.01$, unpaired $t$ test. (D) Left: lysates of macrophages transduced with control LacZ adenovirus or HA CA-CaMKII adenovirus were immunoblotted for HA, LXR $\alpha$, MerTK, and $\beta$-actin. Right: relative density of immunoblot bands for LXR $\alpha$ and MerTK to $\beta$-actin were plotted as relative to LacZ control. Data are shown as mean + SEM. $n=3$ samples per group. ${ }^{*} P<0.05,{ }^{* *} P<0.01$, unpaired $t$ test. (E) Macrophages transduced with either LacZ or HA CA-CaMKII for 60 hours were incubated for 45 additional minutes with a 3:1 ratio of PKH67-green linker-labeled apoptotic Jurkat cells to macrophages and assayed for efferocytosis by image analysis of fluorescent microscopy images. Data are shown as mean $+\mathrm{SEM} . n=3$ sets of macrophages. ${ }^{*} P<0.05$; unpaired $t$ test.

ticular, can trigger the production of proresolving mediators by macrophages $(17,41,50,51)$. Indeed, increasing lesional MerTK by preventing its degradation not only suppresses plaque progression, but also increases resolution mediators in atherosclerotic lesions (39). The possible role of excessive macrophage CaMKII activation in the impaired resolution response in atherosclerosis will be an important topic for future investigation.

Another feature of M-CaMKII-KO lesions is the decreased expression of key markers within the PERK and IRE1 branches of the UPR (Supplemental Figure 4, A-C). Data from the literature and our own laboratory suggest at least 2 links between the decrease in the UPR in M-CaMKII-KO lesions and the decrease in advanced atherosclerosis. First, suppression of the UPR may decrease lesional cell apoptosis (above). For example, genetic targeting of $\mathrm{CHOP}$ in atherosclerosis-prone mice leads to less lesional apoptosis and smaller necrotic cores $(22,52,53)$, as does treatment with an IRE1 inhibitor or with ER stress-relieving "chemical chaperones" (26, 54-56). Moreover, higher expression of CHOP in human coronary artery sections is associated with symptomatic, necrotic plaques (25). Data suggest that the major proatherogenic effect of CHOP is related to apoptosis induction, as CHOP deficiency does not improve efferocytosis (22), but other links between ER stress and impaired efferocytosis are possible (57). Second, suppression of the UPR in M-CaMKII-KO mice may lower lesional inflammation. For example, when the UPR is activated by cholesterol loading of macrophages, elevated CHOP drives Erk1/2-dependent production of IL-6 (58). Moreover, hematopoietic deficiency of CHOP in a mouse model of colitis led to a less severe inflammatory response, at least in part due to a reduction in IL-6 (59). In the context of these two reports, it is interesting to note that the cytokine mRNA showing the greatest reduction in $\mathrm{M}-\mathrm{CaMKII}-\mathrm{KO}$ lesions and macrophages was Il6 (Supplemental Figure 4, D-I). Finally, the ER stress that occurs in advanced lesions may actually promote CaMKII activation, which would create a pathologic amplification loop. This idea is based on the finding that induction of ER stress in cultured macrophages activates CaMKII (16). While we have shown that the PERK and IRE1 branches of the UPR are less active in the M-CaMKII-KO lesions, the expression of ATF6 is concomitantly increased. Although this may seem counterintuitive, there is a precedent for this pattern of UPR activation, both in our work and 
that of others (13-15, 60-63). In this context, ATF6 may serve to regulate the PERK and IRE1 branches of the UPR in order to modulate the response to chronic stress (64).

We have identified ATF6 as a transcriptional regulator of LXR $\alpha$, and our mechanistic and in vivo data are consistent with a model in which LXR $\alpha$ mediates the increase in MerTK-mediated atherosclerotic lesional efferocytosis and the suppression of plaque necrosis when CaMKII is deleted in macrophages. A previous study demonstrating that LXR can induce MerTK provided us with a clue as to how CaMKII deficiency and ATF6 might be linked to MerTK/efferocytosis (28). This study showed that macrophages from LXR $\alpha / \beta$ double-knockout mice had reduced expression of MerTK and impaired efferocytosis (28). In addition, efferocytosis itself activated LXR, perhaps through changes in cellular lipids (28), and another study reported that MerTK signaling can directly activate LXR (65). Thus, there may exist a positive feedback loop between efferocytosis and LXR. Finally, mice treated with LXR agonists have been shown to have both decreased lesion area and smaller necrotic cores $(66,67)$, which is consistent with these previous studies and the new findings herein.

Another possible link between CaMKII and impaired LXR action was suggested by a prior study showing that phosphorylation of LXR $\beta$ by CaMKII abrogated LXR-mediated transrepression of inflammatory genes in several cell lines (68). In addition, CaMKII can phosphorylate components of the nuclear corepressor (NCoR) complex directly, leading to derepression of inflammatory genes (68). In this model, treatment of macrophages with the CaMKII inhibitor KN93 led to increased LXR-mediated transrepression and suppression of inflammation, suggesting that in the absence of active CaMKII, LXR acts unopposed to interact with the NCoR complex. While we have not examined this pathway in lesional macrophages, it may represent a complementary mechanism whereby CaMKII antagonizes LXR activity and thereby promotes lesional inflammation.

In summary, the present study elucidates a role for macrophage CaMKII in the development of advanced atherosclerotic plaque features that are clinically important in humans. We have shown that increased activation of CaMKII in lesional macrophages is associated with advanced and symptomatic atherosclerotic disease in both humans and mice and that deletion of myeloid CaMKII $\gamma$ in atheroprone mice suppresses development of these advanced plaque characteristics. When considered together with the possible roles of CaMKII in lesional smooth muscle cells $(69,70)$, overactive hepatocyte CaMKII in obesity-associated insulin resistance and type 2 diabetes (13-15), and the role of overactive CaMKII in cardiomyocytes in heart failure (12), our findings suggest the potential of targeting this common upstream pathway as an integrated therapeutic approach to cardiometabolic disease.

\section{Methods}

Mice. Ldlr/- mice on the C57BL/6J background (stock 002207) and Lysozyme M-Cre ( $L y s M-C r e)$ mice also on the C57BL/6J background (stock 004781) were purchased from The Jackson Laboratory. Mertk $^{-/-}$mice have been described previously (39). Camk $2 g^{f / f l}$ mice were generated as previously described (39). Camk2fl/fl mice were bred to $L d l r^{/-}$mice to obtain homozygotes for both genes of interest and then subsequently

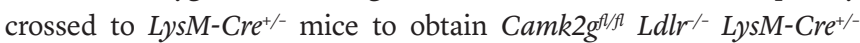

mice and littermate control mice (Camk2 $\left.g^{f / f l} \mathrm{Ldlr}^{\wedge}\right)$. Throughout the manuscript, these mice are referred to as M-CaMKII-KO mice, where $\mathrm{M}$ refers to myeloid, and control mice, respectively. Mice were backcrossed for more than 10 generations to the C57BL/6J background.

Measurement of lipids, insulin, and glucose and complete blood counts. Fasting blood glucose was measured using a glucometer (One Touch Ultra, Lifescan) in mice that were fasted for 4 hours with free access to water. Standard kits were used to measure plasma insulin (Crystal Chem, catalog 90080), plasma triglycerides (Wako, Triglyceride M Kit), and cholesterol (Wako, Cholesterol E kit) per the manufacturer's instructions. Complete blood cell count including leukocyte differential was obtained using a FORCYTE Hematology Analyzer (Oxford Science).

Human plaque histology. The specimens used in the PIT versus TCFA coronary plaque study ( $65 \%$ men, age $71.7 \pm 12.4$ years) were obtained at autopsy. Specimens were formalin fixed, paraffin embedded, and sectioned prior to analysis. Lesions were classified according to Virmani et al. (71). The carotid artery specimens were obtained from patients undergoing carotid endarterectomy for high-grade stenosis of the internal carotid artery at the Division of Vascular Surgery, University Medical Center, Johannes-Gutenberg University. According to preoperative assessment by a consulting neurologist, carotid stenoses were classified as either asymptomatic or symptomatic, defined as TIA or stroke within the past 6 months. After retrieval in the operating room, the excised plaques were rinsed in physiologic saline, immediately snap-frozen in liquid nitrogen, and then transferred to $-80^{\circ} \mathrm{C}$ for storage until processing. Samples were fixed in $4 \%$ formalin overnight, decalcified in EDTA for 4 days, and then embedded in paraffin blocks. Serial sections were obtained at 5- $\mu \mathrm{m}$ intervals. Prior to immunofluorescence staining, all sections from both cohorts were deparaffinized in xylene and then rehydrated in a graded series of ethanol concentrations. Sections were then boiled for 20 minutes in an antigen retrieval buffer (10 mM Tris, 0.5 M EDTA, 0.05\% Tween-20). After rinsing in PBS and blocking in serum-free protein blocking buffer (DAKO, catalog X0909) for 1 hour, sections were incubated overnight at $4^{\circ} \mathrm{C}$ with primary antibodies directed toward p-CaMKII (Novus Biologicals, catalog NB300-184, 1:100), CD68 (DAKO, catalog M0814, 1:100), or SM $\alpha$ A (Sigma-Aldrich, catalog C6198, 1:100). After rinsing in PBS, slides were incubated with secondary antibodies for 2 hours at room temperature. Parallel slides were used for staining with isotype controls. Slides were mounted with DAPI-containing mounting solution, and images were obtained using a Leica microscope and analyzed using FIJI/ImageJ software (NIH). Two slides, each with 2 sections, were assessed for each patient. In parallel, adjacent sections from asymptomatic and symptomatic carotid artery specimens were stained with Harris' H\&E. Total lesion and necrotic core areas were defined as previously described (43).

Mouse atherosclerotic lesion analysis. Eight to ten-week-old male control or M-CaMKII-KO mice were placed on a WD (Envigo, TD 88137 ) for 8,12 , or 16 weeks. Three temporally separate cohorts were structured to include 10 to 14 mice per group with birthdates within 1 to 2 weeks of each other. At the time of harvest, mice were euthanized using isoflurane. Blood was removed by left ventricular puncture, and the vasculature was then perfused with cold PBS. Aortic roots were placed in OCT and immediately frozen or fixed in paraformaldehyde and paraffin embedded. Serial $6-\mu \mathrm{m}$ (paraffin) or 8- $\mu \mathrm{m}$ (frozen) sections were obtained for analysis. For morphometric analysis, 6 paraf- 
fin sections $60 \mu \mathrm{m}$ apart were stained with Harris' H\&E. Total lesion and necrotic core areas were defined as previously described (43). Collagen staining was performed using picrosirius red (Polysciences, catalog 24901A) per the manufacturer's instructions. Collagen cap thickness was quantified from 3 distinct regions of the plaque as previously described (43). TUNEL staining was performed using a kit per the manufacturer's instructions (Roche, catalog 12156792910). For immunofluorescence staining, frozen sections were fixed in cold acetone for 10 minutes. After blocking for 1 hour in serum-free protein blocking buffer (DAKO, catalog X0909), sections were incubated overnight at $4^{\circ} \mathrm{C}$ with the following primary antibodies: Mac3 (BD Biosciences - Pharmingen, catalog 550292, 1:100), SM $\alpha$ A (Sigma-Aldrich, catalog C6198, 1:100), CD3 (Abcam, catalog ab16669, 1:200), Foxp3 (eBioscience, 14-5773-82, 1:200), vWF (Abcam, catalog ab11713, 1:200), p-CaMKII (Novus Biologicals, catalog NB300-184, 1:100), CaMKII $\gamma$ (Novus Biologicals, catalog NBP2-15685, 1:100), or cleaved caspase-3 (Cell Signaling Technologies, catalog 9661, 1:100). Two slides, each with 2 sections, were assessed for each mouse. Parallel slides were used for staining with isotype controls. After rinsing in PBS, slides were incubated with secondary antibodies for 2 hours at room temperature. Slides were counterstained with DAPI, and data were quantified as the percentage of cells positive for markers of interest out of total $\mathrm{DAPI}^{+}$nuclei. All images were captured using a Zeiss fluorescence microscope and analyzed using ImageJ by an observer blinded to the group assignment of each sample.

In situ zymography for collagenase activity. Frozen sections were warmed to $37^{\circ} \mathrm{C}$ and incubated with $20 \mu \mathrm{g} / \mathrm{ml}$ solution of DQ collagen IV (Invitrogen, catalog D12052) or DQ collagen I (Invitrogen, cata$\log \mathrm{D} 12060)$ at $37^{\circ} \mathrm{C}$ overnight. Slides were washed in PBS and then mounted with DAPI-containing solution. Images were captured using a Zeiss fluorescence microscope and analyzed using FIJI/ImageJ by an observer blinded to the group assignment of each sample.

In situ efferocytosis. Experimentation was carried out as previously described by our laboratory $(10,72)$. In brief, aortic root sections were stained with TUNEL (Roche) followed by Mac-3 (BD Biosciences Pharmingen) to label lesional macrophages. Apoptotic cells were then determined to be either macrophage associated (colocalizing or juxtaposed with macrophages) or free (not associated with macrophages). Data were plotted as a ratio of associated to free cells to represent efferocytosis efficiency. Images were captured using a Zeiss fluorescence microscope and analyzed using FIJI/Image J by an observer blinded to the group assignment of each sample.

LCM. Aortic root lesional material from 5 mice was captured (twelve 5 - $\mu \mathrm{m}$ sections per mouse) using a PALM LCM microscope as previously described (72). RNA was isolated using the RNeasy Mini Kit (QIAGEN, catalog 74106) and linearly amplified using the Message Amp II aRNA Kit (Ambion, catalog AM1751). Purity of RNA was estimated by measuring absorbance at 260 and $280 \mathrm{~nm}$ using a NanoDrop (ThermoScientific), and RNA with an $\mathrm{A}_{260 / 280}$ greater than 1.8 was used for cDNA synthesis. cDNA was synthesized using the SuperScript VILO cDNA Synthesis Kit (Invitrogen, catalog 11754050), and RT-qPCR was conducted using a 7500 Real-Time PCR System (Applied Biosystems) and SYBR Green reagents (Applied Biosystems). Specific primer sets used were as follows: Abca1 forward AAAACCGCAGACATCCTTCAG, Abca1 reverse CATACCGAAACTCGTTCACCC; Atf6 forward TGGGTTCGGATATCGCTGTG, Atf6 reverse TGGACTTGGGACTTTGAGCC; Ddit3 (CHOP) forward CTGGAAGCCTGGTATGAGGAT, Ddit3 (CHOP) reverse
CAGGgtCAAGAGTAGTGAAGgT; Dnajb9 forward CCCCAGTGTCAAACTGTACCAG, Dnajb9 reverse AGCGTTTCCAATTTTCCATAAATT; Nr1h3 (LXR $\alpha$ ) forward ATCGCCTTGCTGAAGACCTCTG, Nr1h3 (LXR $\alpha)$ reverse GATGGGGTTGATGAACTCCACC; Nr1h2 (LXR $\beta$ ) forward CCCCACAAGTTCTCTGGACACT, Nr1h2 (LXR $\beta$ ) reverse TGACGTGGCGGAGGTACTG; Mertk forward CAGGGCCTTTACCAGGGAGA, Mertk reverse TGTGTGCTGGATGTGATCTTC; Illb forward GCAACTGTTCCTGAACTCAACT, Illb reverse ATCTTTTGGGGTCCGTCAACT; Il6 forward TAGTCCTTCCTACCCCAATTTCC, Il6 reverse TTGGTCCTTAGCCACTCCTTC; Tnfa forward CTTCTGTCTACTGAACTTCGGG, Tnfa reverse CAGGCTTGTCACTCGAATTTTG; and spliced Xbp1 forward AAGAACACGCTTGGGAATGG, spliced Xbp1 reverse CTGCACCTGCTGCGGAC.

Quantitative PCR. RNA was isolated from macrophages using an RNeasy Kit (QIAGEN), and cDNA was synthesized from RNA (ranging from 500 ng to $1 \mu$ g per reaction) using a cDNA synthesis kit (Applied Biosystems, catalog 4374966). Real-time PCR was performed using a 7500 Real-Time PCR System and SYBR Green reagents (Applied Biosystems). Mouse primer sets used are listed above. Human primer sets used were as follows: $C A M K 2 G$ forward AACAAAAACAGTCTCGTAAGCCC, CAMK2G reverse GCCCTTGATCCCATCTGTAGC (recognizes both mouse and human sequences); NR1H3 (LXR $\alpha$ ) forward AAGCCCTGCATGCCTACGT, NR1H3 reverse TGCAGACGCAGTGCAAACA; and MERTK forward CTCTGGCGTAGAGCTATCACT, MERTK reverse AGGCTGGGTTGGTGAAAACA. Commercially available human ATF6 primers were used (QIAGEN, catalog PPH20143A-200).

Murine bone marrow-derived macrophage culture. Mice were euthanized with isoflurane, and hind legs were removed. Femurs and tibias were flushed with DMEM containing $4.5 \mathrm{~g} / 1$ glucose, $20 \% \mathrm{~L}$-cell media, $10 \%$ HI-FBS, and $1 \%$ penicillin/streptomycin using a 26 -gauge needle. Cell suspensions were passed over a $40-\mu \mathrm{m}$ filter, centrifuged at $500 \mathrm{~g}$, resuspended in $50 \mathrm{ml}$ of media, and plated into five $100-\mathrm{mm}$ dishes. Cells were incubated for 4 days, after which nonadherent cells and debris were aspirated and medium was replaced with fresh medium. After 7 to 10 days of differentiation, with media changed every 2 to 3 days, cells were harvested for use in various experiments.

Human monocyte-derived macrophage culture. Human leukocytes were isolated from buffy coats of deidentified healthy donors (New York Blood Center, New York, New York, USA). In brief, buffy coat was gently layered onto Histopaque solution (Sigma-Aldrich, catalog 10771) and centrifuged at 1,620 $g$ for 25 minutes at room temperature. Leukocytes were removed from the middle layer, washed with RPMI containing 10\% FBS and 1\% penicillin/streptomycin, and then centrifuged at 1,620 $g$ for 5 minutes. This wash step was repeated once before resuspending the pellet in RPMI media and plating cells into 12-well plates. After approximately 3 to 4 hours, when more than $50 \%$ of the cells were adherent, the medium was changed to RPMI containing $10 \%$ FBS, $1 \%$ penicillin/streptomycin, and $10 \mathrm{ng} / \mathrm{ml} \mathrm{GM-CSF}$ (PeproTech, catalog 300-03). Medium was replaced with fresh GM-CSF-containing medium every 2 days. Cells were used for experiments between days 7 and 14, when cells were more than $80 \%$ confluent.

Immunoblotting. Whole cell lysates were harvested in $2 \times$ Laemmli buffer. Nuclear extracts for the detection of activated/cleaved ATF6 were prepared using a kit per the manufacturer's instructions (Active Motif, catalog 40010). Cell extracts were electrophoresed 
on $4 \%-20 \%$ gradient SDS-polyacrylamide gels and transferred to $0.45-\mu \mathrm{m}$ nitrocellulose membranes. Blots were blocked in Tris-buffered saline with $0.1 \%$ Tween-20 containing either $5 \%$ BSA or nonfat milk at room temperature for 1 hour. Membranes were then incubated overnight at $4^{\circ} \mathrm{C}$ with primary antibodies, which included those for ABCA1 (Novus Biologicals, catalog NB400-105, 1:1000), ATF6 (Novus Biologicals, catalog NBP1-40256, 1:500), DACH1 (Proteintech, catalog 10914-1-AP, 1:1000), HDAC4 (Cell Signaling Technologies, catalog 2072, 1:1000), phospho-HDAC4 (Cell Signaling Technologies, catalog 3424S, 1:1000), LXR $\alpha$ (Abcam, catalog ab41902,1:2000), nucleophosmin (Cell Signaling Technologies, cat$\operatorname{alog} 3542,1: 1000$ ), or $\beta$-actin (HRP conjugate, Cell Signaling Technologies, catalog 5125, 1:5000).

Transfection of siRNAs. Bone marrow-derived macrophages were plated at $0.5 \times 10^{6}$ cells/well in a 24-well plate and allowed to adhere overnight. Commercially available siRNAs for CaMKII $\gamma$ (QIAGEN), ATF6 (QIAGEN), and LXR $\alpha$ (QIAGEN) were used. siRNAs were prepared for transfection using Lipofectamine RNAiMax (Invitrogen, catalog 13778-150) per the manufacturer's instructions. Cells were harvested or used for further experiments 36 to 72 hours after transfection as noted in figure legends.

LPS stimulation of bone marrow-derived macrophages. Control and CaMKII-KO bone marrow-derived macrophages were plated at $0.2 \times$ $10^{6}$ cells per well of a 24 -well plate and allowed to adhere overnight. The following day, cells were exposed to medium containing $1 \mathrm{ng} / \mathrm{ml}$ LPS (Sigma-Aldrich, catalog L4391) or vehicle control. After incubating for 4 hours, cells were harvested for mRNA analysis.

In vitro efferocytosis. Bone marrow-derived macrophages were plated at $1 \times 10^{6}$ cells/well in a 12-well plate and allowed to adhere overnight. In some experiments, cells were preincubated with KN92 (Calbiochem, catalog 422709) or KN93 (Calbiochem, catalog 422708). Jurkat cells were labeled with PKH67-GL ( $2 \mu \mathrm{M}$; Sigma-Aldrich) per the manufacturer's instructions, resuspended in full medium at a density of $3 \times 10^{6}$ cells $/ \mathrm{ml}$, and exposed to UV light ( $254 \mathrm{~nm}$, UVP) for $5 \mathrm{~min}$ utes to induce apoptosis. After UV exposure, cells were incubated in a $37^{\circ} \mathrm{C}$ incubator with $5 \% \mathrm{CO}_{2}$ for 1 hour. We routinely obtained approximately $80 \%$ early ACs (annexin V+ PI-) using this procedure. Macrophage medium was replaced with medium containing Jurkat cells to achieve a cell ratio of 3:1 Jurkat cells/macrophages. After incubation for 45 minutes at $37^{\circ} \mathrm{C}$, the medium was aspirated and the macrophages were washed twice with cold PBS. Images were obtained using a Nikon fluorescent microscope and analyzed using FIJI/ImageJ to quantify the percentage of macrophages labeled with PKH67-tagged ACs.

Flow cytometry. Cells were suspended in FACS staining buffer (PBS containing $2 \%$ FBS) at an approximate density of $1 \times 10^{6}$ cells $/ 100 \mu \mathrm{l}$ and preincubated with Fc block for 20 minutes on ice. Cells were then labeled with FITC-F4/80 (eBioscience, catalog 11-4801-82) and biotinylated anti-MerTK (R\&D Systems, catalog BAF591) or isotype controls for 60 minutes on ice. Cells were washed and then incubated with secondary antibody directed at streptavidin for 45 minutes on ice. Cells were washed in FACS buffer and then resuspended for analysis on a BD FACS Canto II. Data were analyzed using FlowJo software.

Confocal microscopy for MerTK staining. Frozen sections were prepared as above. Sections were fixed in cold acetone for 5 minutes, washed in PBS, and then incubated in biotin-blocking buffer (Thermo Scientific, catalog E21390) for 1 hour. Sections were then incubated with biotinylated anti-MerTK (R\&D Systems, catalog BAF591) at $4^{\circ} \mathrm{C}$ overnight, followed by incubation with Alexa Fluor 488-conjugated streptavidin for 1 hour. Slides were mounted with DAPI-containing solution, viewed on a Nikon A1 confocal microscope, and analyzed using FIJI/ImageJ software.

ChIP. Confluent 10-cm dishes of bone marrow-derived macrophages (1 dish per experimental condition) from each genotype were prepared for use on days 7 to 10 of differentiation. Cells were washed with cold PBS containing protease inhibitors and then incubated with $2 \mathrm{mM}$ disuccinimidyl glutarate in PBS for 30 minutes at room temperature. Paraformaldehyde (PFA) was added to a final concentration of $1 \%$ before incubating for an additional 15 minutes at room temperature. Crosslinking was terminated by incubating cells in $200 \mathrm{mM}$ Tris- $\mathrm{HCl} \mathrm{pH}$ 8 followed by $100 \mathrm{mM}$ Tris-HCl pH 8, each for 15 minutes. Cells were then harvested in $1 \mathrm{ml}$ of cold PBS containing protease inhibitors and pelleted by centrifugation at $2000 \mathrm{~g}$ for 5 minutes at $4^{\circ} \mathrm{C}$. Pellets were resuspended in $1 \mathrm{ml}$ of nuclei isolation buffer ( $50 \mathrm{mM}$ Tris- $\mathrm{HCl} \mathrm{pH} \mathrm{8,} 60$ $\mathrm{mM} \mathrm{KCl}, 0.5 \% \mathrm{NP}-40,1 \times$ protease inhibitors) and incubated on ice for 10 minutes. Samples were again centrifuged at $2000 \mathrm{~g}$ for 5 minutes at $4^{\circ} \mathrm{C}$, after which pellets were resuspended in $130 \mu \mathrm{l}$ lysis buffer $(50 \mathrm{mM}$ Tris-HCl pH 8, 0.5\% SDS, 10 mM EDTA pH 8, 0.5 mM EGTA pH 8) and transferred to Covaris microtubes (catalog 520045). DNA was sheared to an average size of 400-500 bps using a Covaris S2 focused ultrasonicator set at duty 5\%, intensity 4, cycles 200, 15 seconds for 12 total repetitions. After sonication, samples were centrifuged at maximum speed for 10 minutes at $4^{\circ} \mathrm{C}$ and supernatants were harvested to fresh tubes. Fragmented chromatin $(25 \mu \mathrm{l})$ was removed for use as input. These samples were treated with elution buffer $\left(0.1 \mathrm{M} \mathrm{NaHCO}_{3}, 1 \%\right.$ SDS, $0.2 \mathrm{M}$ $\mathrm{NaCl}$, and $0.25 \mu \mathrm{g} / \mathrm{ml} \mathrm{RNase} \mathrm{A)} \mathrm{and} \mathrm{incubated} \mathrm{for} 30$ minutes at room temperature to digest RNA, followed by incubation at $65^{\circ} \mathrm{C}$ for 4 hours to reverse protein crosslinks. Samples were then treated with proteinase $\mathrm{K}$ in $100 \mu \mathrm{M} \mathrm{EDTA} / 6.5 \mathrm{mM}$ Tris- $\mathrm{HCl} \mathrm{pH} 8$ for 1 hour at $45^{\circ} \mathrm{C}$. The resulting products were purified using a QIAquick PCR Purification Kit (QIAGEN, catalog 28106) and quantitated using a NanoDrop spectrophotometer. For each sample, $200 \mathrm{ng}$ was run on an agarose gel to ensure that even and adequate sonication of chromatin was obtained. The remaining 105 $\mu \mathrm{l}$ of the sheared chromatin sample was diluted to $200 \mu \mathrm{l}$ in lysis buffer and then further diluted to a final volume of $1 \mathrm{ml}$ in dilution buffer (1\% Triton X-100, 2 mM EDTA pH 8, 150 mM NaCl, 20 mM Tris-HCl pH 8, $1 \times$ protease inhibitors). These samples were then precleared by incubating with $0.25 \mu \mathrm{g}$ of normal mouse IgG per $1 \mu \mathrm{g}$ of chromatin for 1 hour with end-over-end rotation at $4^{\circ} \mathrm{C}$, followed by the addition of $40 \mu \mathrm{l}$ of prewashed magnetic beads (Dynabeads, Invitrogen, catalog 10004D) for an additional hour. Using a magnetic rack to pellet the beads, the supernatant from each sample was removed and divided into two 500- $\mu \mathrm{l}$ aliquots. Each aliquot was brought to a final volume of $1 \mathrm{ml}$ with dilution buffer, and then $1 \mu \mathrm{g}$ of either normal mouse IgG or mouse anti-ATF6 antibody (Novus Biologicals, catalog NBP1-40256) was added to each 5 $\mu \mathrm{g}$ of chromatin. Samples were rotated end-over-end overnight at $4^{\circ} \mathrm{C}$, then centrifuged at maximum speed for 10 minutes at $4^{\circ} \mathrm{C}$. Supernatants were transferred to fresh tubes, $40 \mu$ of beads was added, and samples were then incubated for 2 hours with rotation at $4^{\circ} \mathrm{C}$. After immunoprecipitation, beads were washed once for 5 minutes with wash buffer no. 1 (20 mM Tris-HCl pH 7, 150 mM NaCl, 0,1\% SDS, 1\% Triton X-100, 2 mM EDTA pH 8), then once with wash buffer no. 2 (10 mM Tris-HCl pH 7, $250 \mathrm{mM} \mathrm{LiCl}, 1 \% \mathrm{NP}-40$, 0.7\% sodium deoxycholate, $1 \mathrm{mM}$ EDTA pH 8), and then finally with Tris-EDTA buffer $\mathrm{pH}$ 8. After the final wash, samples were treated with elution buffer containing RNase A and then 
proteinase $\mathrm{K}$, and the samples were purified for PCR as described above. The presence of a Nr1h3 (LXR $\alpha)$ intronic region in the anti-ATF6 precipitated samples was quantified by qPCR and expressed relative to the input genomic DNA (Nr1h3 specific primers: forward ATCTGGGCGGGGATAGAGTT, reverse GGCAGAACCACAAGACTGGA). Primers to a region of the Nr1h3 gene that does not contain the consensus ATF6 site were used to confirm that no nonspecific binding of the ATF6 antibody was obtained (nonspecific Nr1h3 primers: forward GGCTAGGGGGAATGAGATGC, reverse TGCTCCCAATAGAGGACCCA).

Viral transduction. Bone marrow-derived macrophages were plated at $0.2 \times 10^{6}$ cells/well in a 24 -well plate and allowed to adhere overnight. High-titer $\left(5 \times 10^{6} \mathrm{pfu} / \mathrm{ml}\right)$ adenovirus containing either CA-CaMKII or LacZ was produced by Viraquest Inc. based on previously described vectors $(15,32)$ that were a gift of Harold Singer (Albany Medical Center). Macrophages were transduced with 200 MOI of LacZ or CA-CaMKII virus and harvested for mRNA or protein 60 hours later.

Statistics. All results are represented as mean + SEM. Normality was determined using D’Agostino-Pearson and/or Shapiro-Wilk normality testing. $P$ values for normally distributed data were calculated using either Student's $t$ test or 2-way ANOVA with post hoc Tukey's analysis. $P$ values for nonnormally distributed data were calculated using the Mann-Whitney $U$ test. For the data in Figure $1 \mathrm{~B}$ as well as Supplemental Figure 1, A and B, correlation coefficients $(r)$ and $P$ values were calculated using Pearson product-moment correlation analysis.

Study approval. For human studies, carotid endarterectomy samples were obtained from coauthor BD. Coronary artery samples were obtained from coauthor JCS. The specimens used in the PIT versus TCFA coronary plaque study were obtained at autopsy, with prior consent and in agreement with the code for proper secondary use of human tissue in the Netherlands (http://www.fmwv.nl). All samples were obtained with consent from patients. Use of all material conformed to the Declaration of Helsinki and was approved by the appropriate university ethics review boards. For animal studies, all mice were cared for according to NIH and
IACUC guidelines in a barrier facility at Columbia University Medical Center, and procedures involving mice were performed with the approval of the Columbia University Medical Center IACUC.

\section{Author contributions}

ACD, LO, GF, and IT conceived and designed the research. BD and JCS donated patient samples and were instrumental in the interpretation of the human data. ACD, LO, BC, ZZ, CCR, GK, and GF conducted the experiments. ACD, LO, BC, ZZ, GF, JH, ART, and IT analyzed the data. ACD and IT wrote the paper.

\section{Acknowledgments}

The authors would like to thank Johannes Backs and Eric N. Olson for providing Camk2 $g^{f l / f l}$ mice, Harold Singer for providing the CA-CaMKII adenovirus, and Chris Glass and Nathan Spann for advice on the ChIP experiments. This work was supported by NIH Cardiology Fellowship T32 training grant HL007854-21 (to ACD), NIH grant DK106045 (to LO), Pilot and Feasibility grant from the Columbia University Medical Center Diabetes Research Center P30 DK063608 (to LO), an American Heart Association postdoctoral fellowship grant (to BC), Berrie Scholars Award UR007997 from The Russell Berrie Foundation (to ZZ), NIH grant HL119587 (to GF), American Federation for Aging Research Grant for Junior Faculty A16034 (to GF), and NIH grants HL132412, HL075662, and HL127464 (to IT). These studies used the resources of the Columbia Diabetes and Endocrinology Research Center and Columbia Cancer Center flow core facilities, funded in part through NIH center grants 5P30DK063608 and P30CA013696, respectively.

Address correspondence to: Ira Tabas, $630 \mathrm{~W}$ 168th Street, New York, New York 10032, USA. Phone: 212.305.5669; Email: iat1@ cumc.columbia.edu.
1. Mozaffarian D, et al. Heart disease and stroke statistics--2015 update: a report from the American Heart Association. Circulation. 2015;131(4):e29-322.

2. Glass CK, Witztum JL. Atherosclerosis. The road ahead. Cell. 2001;104(4):503-516.

3. Lusis AJ. Atherosclerosis. Nature. 2000;407(6801):233-241.

4. Hansson GK, Libby P, Tabas I. Inflammation and plaque vulnerability. J Intern Med. 2015;278(5):483-493.

5. Yahagi K, et al. Pathophysiology of native coronary, vein graft, and in-stent atherosclerosis. Nat Rev Cardiol. 2016;13(2):79-98.

6. Tabas I. Macrophage death and defective inflammation resolution in atherosclerosis. Nat Rev Immunol. 2010;10(1):36-46.

7. Schrijvers DM, De Meyer GR, Kockx MM, Herman AG, Martinet W. Phagocytosis of apoptotic cells by macrophages is impaired in atherosclerosis. Arterioscler Thromb Vasc Biol. 2005;25(6):1256-1261.

8. Linton MF, Babaev VR, Huang J, Linton EF, Tao $\mathrm{H}$, Yancey PG. Macrophage apoptosis and efferocytosis in the pathogenesis of atherosclerosis. Circ J. 2016;80(11):2259-2268.
9. Cai B, et al. MerTK receptor cleavage promotes plaque necrosis and defective resolution in atherosclerosis. J Clin Invest. 2017;127(2):564-568.

10. Thorp E, Cui D, Schrijvers DM, Kuriakose G, Tabas I. Mertk receptor mutation reduces efferocytosis efficiency and promotes apoptotic cell accumulation and plaque necrosis in atherosclerotic lesions of apoe-/-mice. Arterioscler Thromb Vasc Biol. 2008;28(8):1421-1428.

11. Anderson ME. Oxidant stress promotes disease by activating CaMKII. J Mol Cell Cardiol. 2015;89(Pt B):160-167.

12. Rokita AG, Anderson ME. New therapeutic targets in cardiology: arrhythmias and $\mathrm{Ca} 2+/ \mathrm{calm}-$ odulin-dependent kinase II (CaMKII). Circulation. 2012;126(17):2125-2139.

13. Ozcan L, Cristina de Souza J, Harari AA, Backs J, Olson EN, Tabas I. Activation of calcium/calmodulin-dependent protein kinase II in obesity mediates suppression of hepatic insulin signaling. Cell Metab. 2013;18(6):803-815.

14. Ozcan L, et al. Hepatocyte DACH1 is increased in obesity via nuclear exclusion of HDAC4 and promotes hepatic insulin resistance. Cell Rep. 2016;15(10):2214-2225.

15. Ozcan L, et al. Calcium signaling through CaM-
KII regulates hepatic glucose production in fasting and obesity. Cell Metab. 2012;15(5):739-751.

16. Timmins JM, et al. Calcium/calmodulin-dependent protein kinase II links ER stress with Fas and mitochondrial apoptosis pathways. JClin Invest. 2009;119(10):2925-2941.

17. Fredman G, et al. Resolvin D1 limits 5-lipoxygenase nuclear localization and leukotriene B4 synthesis by inhibiting a calcium-activated kinase pathway. Proc Natl Acad Sci US A. 2014;111(40):14530-14535.

18. Colbran RJ. Regulation and role of brain calcium/ calmodulin-dependent protein kinase II. Neurochem Int. 1992;21(4):469-497.

19. Toussaint F, Charbel C, Allen BG, Ledoux J. Vascular CaMKII: heart and brain in your arteries. Am J Physiol Cell Physiol. 2016;311(3):C462-C478.

20. Clausen BE, Burkhardt C, Reith W, Renkawitz R, Förster I. Conditional gene targeting in macrophages and granulocytes using LysMcre mice. Transgenic Res. 1999;8(4):265-277.

21. Finn AV, Nakano M, Narula J, Kolodgie FD, Virmani R. Concept of vulnerable/unstable plaque. Arterioscler Thromb Vasc Biol. 2010;30(7):1282-1292.

22. Thorp E, Li G, Seimon TA, Kuriakose G, Ron D, Tabas I. Reduced apoptosis and plaque 
necrosis in advanced atherosclerotic lesions of Apoe $^{-/-}$and Ldlr $/-$mice lacking CHOP. Cell Metab. 2009;9(5):474-481.

23. Libby P. Collagenases and cracks in the plaque. JClin Invest. 2013;123(8):3201-3203.

24. Moore KJ, Tabas I. Macrophages in the pathogenesis of atherosclerosis. Cell. 2011;145(3):341-355.

25. Myoishi M, et al. Increased endoplasmic reticulum stress in atherosclerotic plaques associated with acute coronary syndrome. Circulation. 2007;116(11):1226-1233.

26. Tufanli O, et al. Targeting IRE1 with small molecules counteracts progression of atherosclerosis. Proc Natl Acad Sci U S A. 2017;114(8):E1395-E1404.

27. Ait-Oufella H, et al. Defective mer receptor tyrosine kinase signaling in bone marrow cells promotes apoptotic cell accumulation and accelerates atherosclerosis. Arterioscler Thromb Vasc Biol. 2008;28(8):1429-1431.

28. A-Gonzalez N, et al. Apoptotic cells promote their own clearance and immune tolerance through activation of the nuclear receptor LXR. Immunity. 2009;31(2):245-258

29. ENCODE Project Consortium. A user's guide to the encyclopedia of DNA elements (ENCODE) PLoS Biol. 2011;9(4):e1001046.

30. O'Geen H, Echipare L, Farnham PJ. Using ChIPseq technology to generate high-resolution profiles of histone modifications. Methods Mol Biol. 2011;791:265-286.

31. Ram O, et al. Combinatorial patterning of chromatin regulators uncovered by genomewide location analysis in human cells. Cell. 2011;147(7):1628-1639.

32. Pfleiderer PJ, Lu KK, Crow MT, Keller RS, Singer HA. Modulation of vascular smooth muscle cell migration by calcium/ calmodulin-dependent protein kinase II-delta 2. Am J Physiol Cell Physiol. 2004;286(6):C1238-C1245.

33. Arandjelovic S, Ravichandran KS. Phagocytosis of apoptotic cells in homeostasis. Nat Immunol. 2015;16(9):907-917.

34. Greenlee-Wacker MC, Rigby KM, Kobayashi SD, Porter AR, DeLeo FR, Nauseef WM. Phagocytosis of Staphylococcus aureus by human neutrophils prevents macrophage efferocytosis and induces programmed necrosis. J Immunol. 2014;192(10):4709-4717.

35. Lin J, et al. A role of RIP3-mediated macrophage necrosis in atherosclerosis development. Cell Rep. 2013;3(1):200-210.

36. Tabas I. Heart disease: Death-defying plaque cells. Nature. 2016;536(7614):32-33.

37. Thorp E, Subramanian M, Tabas I. The role of macrophages and dendritic cells in the clearance of apoptotic cells in advanced atherosclerosis. Eur J Immunol. 2011;41(9):2515-2518.

38. Kojima Y, et al. CD47-blocking antibodies restore phagocytosis and prevent atherosclerosis. Nature. 2016;536(7614):86-90.

39. Cai B, et al. MerTK cleavage limits proresolving mediator biosynthesis and exacerbates tissue inflammation. Proc Natl Acad Sci U S A. 2016;113(23):6526-6531

40. Merched AJ, Ko K, Gotlinger KH, Serhan CN, Chan L. Atherosclerosis: evidence for impair- ment of resolution of vascular inflammation governed by specific lipid mediators. FASEB $J$. 2008;22(10):3595-3606.

41. Fredman G, et al. An imbalance between specialized pro-resolving lipid mediators and pro-inflammatory leukotrienes promotes instability of atherosclerotic plaques. Nat Commun. 2016;7:12859.

42. Viola JR, et al. Resolving lipid mediators maresin 1 and resolvin D2 prevent atheroprogression in mice. Circ Res. 2016;119(9):1030-1038.

43. Fredman G, et al. Targeted nanoparticles containing the proresolving peptide Ac2-26 protect against advanced atherosclerosis in hypercholesterolemic mice. Sci Transl Med. 2015;7(275):275ra20.

44. Hasturk H, et al. Resolvin E1 (RvE1) attenuates atherosclerotic plaque formation in diet and inflammation-induced atherogenesis. Arterioscler Thromb Vasc Biol. 2015;35(5):1123-1133.

45. Duffield JS, Lupher M, Thannickal VJ, Wynn TA. Host responses in tissue repair and fibrosis. Annu Rev Pathol. 2013;8:241-276.

46. Scott JA, et al. The multifunctional $\mathrm{Ca}^{2+} /$ calmodulin-dependent kinase II $(\mathrm{CaMKII} \delta$ ) regulates arteriogenesis in a mouse model of flowmediated remodeling. PLoS One. 2013;8(8):e71550

47. Wu CY, Hsieh HL, Sun CC, Yang CM. IL-1beta induces MMP-9 expression via a Ca2+-dependent CaMKII/JNK/c-JUN cascade in rat brain astrocytes. Glia. 2009;57(16):1775-1789.

48. Aikawa M, Libby P. The vulnerable atherosclerotic plaque: pathogenesis and therapeutic approach. Cardiovasc Pathol. 2004;13(3):125-138

49. Gough PJ, Gomez IG, Wille PT, Raines EW. Macrophage expression of active MMP-9 induces acute plaque disruption in apoE-deficient mice. JClin Invest. 2006;116(1):59-69.

50. Serhan CN, Chiang N, Van Dyke TE. Resolving inflammation: dual anti-inflammatory and pro-resolution lipid mediators. Nat Rev Immunol. 2008;8(5):349-361

51. Dalli J, Serhan CN. Specific lipid mediator signatures of human phagocytes: microparticles stimulate macrophage efferocytosis and pro-resolving mediators. Blood. 2012;120(15):e60-e72.

52. Gao J, et al. Involvement of endoplasmic stress protein C/EBP homologous protein in arteriosclerosis acceleration with augmented biological stress responses. Circulation. 2011;124(7):830-839.

53. Tsukano $\mathrm{H}$, et al. The endoplasmic reticulum stress-C/EBP homologous protein pathway-mediated apoptosis in macrophages contributes to the instability of atherosclerotic plaques. Arterioscler Thromb Vasc Biol. 2010;30(10):1925-1932.

54. Dong Y, et al. Reduction of AMP-activated protein kinase alpha2 increases endoplasmic reticulum stress and atherosclerosis in vivo. Circulation. 2010;121(6):792-803.

55. Erbay E, et al. Reducing endoplasmic reticulum stress through a macrophage lipid chaperone alleviates atherosclerosis. Nat Med. 2009;15(12):1383-1391.

56. Zeng L, et al. Sustained activation of XBP1 splicing leads to endothelial apoptosis and atherosclerosis development in response to disturbed flow. Proc
Natl Acad Sci U S A. 2009;106(20):8326-8331.

57. Cash JG, et al. Apolipoprotein E4 impairs macrophage efferocytosis and potentiates apoptosis by accelerating endoplasmic reticulum stress. J Biol Chem. 2012;287(33):27876-27884.

58. Li Y, et al. Free cholesterol-loaded macrophages are an abundant source of tumor necrosis factor-alpha and interleukin-6: model of NFkappaB- and map kinase-dependent inflammation in advanced atherosclerosis. J Biol Chem. 2005;280(23):21763-21772.

59. Datta S, et al. cEBP Homologous protein expression in macrophages regulates the magnitude and duration of IL-6 expression and dextran sodium sulfate colitis. JInterferon Cytokine Res. 2015;35(10):785-794.

60. Engin F, et al. Restoration of the unfolded protein response in pancreatic $\beta$ cells protects mice against type 1 diabetes. Sci Transl Med. 2013;5(211):211ra156.

61. Jin JK, et al. ATF6 decreases myocardial ischemia/reperfusion damage and links ER stress and oxidative stress signaling pathways in the heart. Circ Res. 2017;120(5):862-875.

62. Martindale JJ, et al. Endoplasmic reticulum stress gene induction and protection from ischemia/ reperfusion injury in the hearts of transgenic mice with a tamoxifen-regulated form of ATF6. Circ Res. 2006;98(9):1186-1193.

63. Toko H, et al. ATF6 is important under both pathological and physiological states in the heart. JMol Cell Cardiol. 2010;49(1):113-120.

64. Wu J, et al. ATF6alpha optimizes long-term endoplasmic reticulum function to protect cells from chronic stress. Dev Cell. 2007;13(3):351-364.

65. Choi JY, Seo JY, Yoon YS, Lee YJ, Kim HS, Kang JL. Mer signaling increases the abundance of the transcription factor LXR to promote the resolution of acute sterile inflammation. Sci Signal. 2015;8(365):ra21.

66. Gui Y, et al. A novel small molecule liver X receptor transcriptional regulator, nagilactone $\mathrm{B}$, suppresses atherosclerosis in apoE-deficient mice. Cardiovasc Res. 2016;112(1):502-514.

67. Levin N, et al. Macrophage liver X receptor is required for antiatherogenic activity of LXR agonists. Arterioscler Thromb Vasc Biol. 2005;25(1):135-142.

68. Huang W, et al. Coronin 2A mediates actin-dependent de-repression of inflammatory response genes. Nature. 2011;470(7334):414-418.

69. Maione AS, et al. Cellular subtype expression and activation of CaMKII regulate the fate of atherosclerotic plaque. Atherosclerosis. 2017;256:53-61.

70. Saddouk FZ, Ginnan R, Singer HA. Ca(2+)/calmodulin-dependent protein kinase II in vascular smooth muscle. Adv Pharmacol. 2017;78:171-202.

71. Virmani R, Kolodgie FD, Burke AP, Farb A, Schwartz SM. Lessons from sudden coronary death: a comprehensive morphological classification scheme for atherosclerotic lesions. Arterioscler Thromb Vasc Biol. 2000;20(5):1262-1275.

72. Subramanian M, Thorp E, Hansson GK, Tabas I. Treg-mediated suppression of atherosclerosis requires MYD88 signaling in DCs. JClin Invest. 2013;123(1):179-188. 